\title{
Finite Brand Loyalty and Equilibrium Price Promotions
}

\author{
Bing Jing and Zhong Wen \\ Stern School of Business, New York University \\ June 26, 2005
}

\begin{abstract}
The extant literature on price promotions typically assumes that consumers loyal to a brand never switch to a competing brand, with Shilony (1977) and Raju et al (1990) being exceptions. Extending the Narasimhan (1988) model, we allow loyal consumers to hold finite brand loyalty. Our unique equilibrium splits into three types, depending upon configurations of consumer reservation utility, brand strength and switcher population. The type of equilibrium for high brand loyalty corresponds to the one in Narasimhan (1988). The remaining two types for intermediate and low brand loyalty demonstrate strikingly different properties. First, the strong brand has a higher price range and a higher regular price. Second, the strong brand has a higher (lower) average promotional depth than the weak brand when the switcher population is small (large). Third, both brands promote equally frequently when brand loyalty is relatively low. Therefore, this analysis hopefully provides a more complete picture about firms' promotional decisions for all possible levels of brand loyalty and switcher pupulation. (Key Words: Price promotions; Brand loyalty; Private labels)
\end{abstract}




\section{Introduction}

Marketing researchers usually explain price dispersion through models with mixed-strategy equilibria. In the literature on competitive price promotions, it is typically assumed that each firm has a captive customer base over which it can act as a monopoly, and that all firms also face a common pool of mobile customers for which they may compete on the sole basis of price (e.g., Narasimhan 1988, Colombo and Morrison 1989, McGahan and Ghemawat 1994, Simester 1997, Lal and Villas-Boas 1996, 1998, Chen et al. 2001a, 2001b, Iyer and Pazgal 2003)1. Each firm has its own base of "loyal customers" who are willing to purchase its product at a price not exceeding their reservation utility, and the firm(s) with the lowest price will attract the remaining "switchers" or price shoppers. In these models, therefore, each firm may choose to either serve only its captive consumers at the monopoly price or to serve both the mobile and its own captive consumers at the lowest market price, and the firms' attempt to achieve a balance between these two options results in mixed pricing strategies.

Obviously, such a dichotomous demand specification intends to capture the heterogeneity in consumers' sensitivity to prices and brands. Besides, in the presence of price-sensitive switchers, the requirement that the loyal consumers do not switch also plays a crucial role in ensuring a more tractable analysis $^{2}$. However, the assumption that the loyal consumers are always captive does not appear to reflect accurately the impact of brand loyalty on

\footnotetext{
${ }^{1} \mathrm{~A}$ similar demand structure also frequently appears in the economic literature on consumer search. For example, in Salop and Stiglitz (1977), Varian (1980), Rosenthal (1981), Png and Hirshleifer (1987) and Baye and Morgan (2001), some consumers have prohibitively high search costs and are thus "uninformed", while the remaining consumers have zero search costs and are "informed" about the prices charged by all firms. Each firm secures at least an equal share of business from the uninformed buyers, while the informed buyers only patronize the firm(s) with the lowest price.

${ }^{2}$ In a duopoly model with switchers, each firm's payoff function has only one point of discontinuity (when the two firms' prices are equal) if the loyal consumers can not switch under the absolute-loyalty assumption, but the number of discontinuities becomes two if the loyal consumers can switch under a finite-loyalty assumption to be described shortly.
} 
cross-price elasticity. In many product or service markets, a substantial fraction of the consumers that would remain loyal to a brand under normal circumstances switch to a competing brand when the latter offers a sufficiently deep discount. Amazon.com is often imputed to be the strongest brand in online book retailing, but the price premium it can enjoy is still limited to a few dollars per title (Brynjolfsson and Smith 2000). To our knowledge, the observation of finite brand loyalty has previously been incorporated into the theoretical models by Shilony (1977), Raju et al. (1990) and Rao (1991).

In a duopoly where there are switchers and where the two brands differ in loyal segment size, the present paper examines how finite brand loyalty held by loyal consumers may affect the firms' price promotional decisions. Following Pessemier (1959), Jacoby and Chestnut (1978) and Raju et al (1990), we define the brand strength of a firm as the minimum price discount offered by some competitor to cause the former's loyal customers to defect. Specifically, we consider competition between a strong brand and a weak brand. The strong brand has a segment of loyal customers and the remaining consumers are switchers and not attached to either brand. Thus one may regard the strong brand as a national or well-established brand, and the weak a private label or new brand. Our model allows brand strength to be below consumer reservation utility so that consumers loyal to the strong brand will switch to the weak brand if the latter offers a discount exceeding the former's brand strength.

Therefore, our model has three key elements, namely asymmetric loyal segments, presence of switchers and finite brand loyalty. Our model thus combines the main features of three well-known models in the promotions literature: Narasimhan (1988) (for asymmetric loyal segments and presence of switchers), Shilony (1977) and Raju et al. (1990) (for finite brand loyalty). We next compare with each of the three in greater detail.

(A) Narasimhan (1988). Assuming that consumers loyal to a brand never switch, Narasimhan (1988) presents a simple and yet elegant model 
consisting of two brands with asymmetrically-sized loyal segments and a segment of switchers. He focuses on how asymmetric loyal segments drive different pricing strategies. His major results include: (1) Both firms price in the same continuous range and thus have the same regular price ${ }^{3} ;(2)$ The strong brand promotes less frequently than the weak brand; and (3) On average, the strong brand charges a higher price, but the two brands offer discounts of the same depth.

Compared with Narasimhan (1988), our model retains the presence of switchers and asymmetry in loyal segment size, but allows brand loyalty to fall below consumer reservation utility ${ }^{4}$. The unique mixed-strategy equilibrium in our model divides into three types corresponding to high, intermediate and low brand strength, respectively. The original equilibrium in Narasimhan (1988) emerges as the type for high brand strength, where it is feasible but not profitable for the weak brand to attract the loyal consumers of the strong brand. The two types of equilibria for intermediate and low brand strength demonstrate strikingly different properties than the one for high brand strength. First, the price range of the strong brand is always higher than that of the weak brand, in that both the maximum and minimum prices of the former are greater than those of the latter, respectively ${ }^{5}$. If we interpret a brand's maximum price as its regular price, then the strong

\footnotetext{
${ }^{3}$ The result that both firms have the same price range does not seem sensitive to the asymmetry in loyal segments. In Varian (1980), firms with symmetric shares of the "uninformed" (and thus loyal) consumers also have identical price ranges in equilibrium.

${ }^{4}$ Since we normalize the loyal segment of the weak brand to zero, one may alternatively view the loyal segment of the strong brand as the size difference between the two brands' loyal segments.

${ }^{5}$ In our model the two brands are homogeneous in that they command identical consumer reservation utility. Narasimhan (1988) also considers the case of differentiated products in the sense that all consumers hold a higher reservation utility for one brand than for the other (see pages 439-440), and shows that the better valued brand has a higher price range. However, there the mechanism for generating asymmetric price ranges is fundamentally different than ours. Since in his model each brand's loyal consumers are its captive demand, each brand always prices up to its reservation utility. Thus the price range of the better-valued brand is higher than that of the other brand by precisely the difference in their reservation utilities.
} 
brand charges a higher regular price. Second, the average promotional depth of the strong brand is higher (lower) than that of the weak brand when the population of switchers is small (large). Third, when brand strength is low enough, neither brand's strategy has a mass point, i.e., both brands may promote equally frequently. Fourth, the mean prices of both sellers are unimodal functions of the switcher population.

The differences between these two types of equilibria and Narasimhan's arise from our assumption of finite, instead of absolute, brand loyalty. For low and intermediate values of brand loyalty, in equilibrium consumers loyal to the strong brand switch to the weak brand with positive probability. In Narasimhan (1988), as the switcher population grows, even the strong brand will have a greater incentive to attract the large chunk of switchers, thus intensifying competition. This force is still present in our model. However, assuming finite brand loyalty introduces a new force: When the switcher population decreases, the weak brand has a stronger incentive to attract its rival's loyal customers by offering deep discounts, also intensifying competition. Therefore, in these two types of equilibria price competition is most intense (relaxed) for extreme (intermediate) sizes of switcher population.

(B) Shilony (1977). Common to Shilony (1977) and our current model is that both allow brand strength to fall below consumer reservation utility. His symmetric equilibrium also divides into three types according to the magnitude of brand strength. For high brand strength, a pure-strategy equilibrium obtains at the monopoly price ${ }^{6}$. For intermediate and low values of brand strength, mixed-strategy equilibria obtain, i.e., there is price dispersion. In particular, for low values of brand strength, the mixed strategy has no mass points and competition is so fierce that no firm can price up to consumer reservation utility.

Otherwise, Shilony's model is very different than ours in that he examines a totally symmetric setting with no switchers. In his model, the firms

\footnotetext{
${ }^{6}$ This is due to the absence of price-sensitive switchers in his model.
} 
have equally-sized loyal segments. In our model, two brands with asymmetric loyal segment sizes compete in the presence of switchers. We choose to include switchers because in many markets there exists a non-negligible fraction of consumers that are more sensitive to price than to brand name. Consequently, our equilibrium has the following different features. Due to the presence of switchers, all three types of equilibria demonstrate price dispersion. The two brands may have asymmetric strategy sets. In particular, each firm's price range (bounded by brand strength) is narrower than that in Shilony (1977), where the price range is bounded by twice the brand strength (except for the case of pure-strategy equilibrium). In addition, our results on promotional frequency and depth are unique to the asymmetric setting.

As mentioned earlier, much of the extant literature adopts a dichotomized demand specification. In comparison, Shilony's approach may be viewed as a compromise: there is only one group of consumers that are partially captive to each firm; they can switch among the brands at some cost. Our model still admits two distinct consumer groups, one partially captive to each brand and the other completely mobile.

(C) Raju (1988) and Raju et al. (1990). Raju (1988) and Raju et al. (1990) extend Shilony (1977) to an asymmetric setting by allowing different brands to command different degrees of consumer loyalty. As in Shilony (1977), there are no switchers and each of the two firms has its own loyal segment in these two papers. Raju's model assumes that the strong brand can always attract consumers loyal to the weak brand, and separately considers two scenarios depending on whether the weak brand can also attract consumers loyal to the strong brand. In both scenarios, Raju finds that the strong brand promotes less often and that the two brands have the same regular price (at consumer reservation utility) (Chapters 2 and 3 of Raju 1988, Propositions 6 and 11 of Raju et al. 1990). When brand disparity is large so that the weak brand can not profitably attract those loyal to the strong brand (whether the two loyal segments are equally sized or not), he 
also finds that the strong brand offers a higher average discount and that the lowest price of the strong brand is below that of the weak brand. When the two loyal segments are equally-sized and brand disparity is small so that the weak brand can also sell to those consumers loyal to the strong brand, Raju finds that it is the weak brand that offers a larger discount (Chapter 4 of Raju 1988, Proposition 12 of Raju et al 1990). Therefore, his result on promotional depth is contingent on the relative strength between the two brands. In addition, the strategies of both brands always have a mass point at consumer reservation utility (due to the absence of switchers), and the strategy set of the strong brand always contains a gap.

As in Raju's model, we consider competition between two brands with finite brand loyalty. However, our model has two key differences. First, the distinction between the strong and weak brands is made on the basis of loyal segment size in our model (and also Narasimhan's), but is made on the basis of relative brand strength in Raju's papers. Second, our model contains switchers, while Raju's model does not. Consequently, our model reveals some different promotional dynamics. The regular and lowest prices of the strong brand are always at or higher than those of the weak brand. Our result on promotional depth is also very different. When brand strength is high so that the weak brand can not attract consumers loyal to the strong brand, both brands offer the same average discount. Otherwise, the strong brand offers a higher (lower) average discount than the weak brand when the switcher population is small (large). Therefore, we find that when brand strength is not very high so that the weak brand can attract the loyal consumers of the strong brand, which brand offers higher discounts depends on the population of switchers. In our model, the weak brand's strategy never has a mass point and the strong brand's strategy set never contains a gap.

Our result on promotional frequency is basically consistent with Raju's. We show that the strong brand promotes less often in two of the three types of equilibria, and that both brands promote equally frequently when brand 
strength is relatively low.

In addition, Rao (1991) also allows brand loyalty to fall below consumer reservation utility. Unlike the other models, Rao (1991) assumes that the loyal consumers have different, continuous grades of brand loyalty. The plus side of this treatment is its proximity to reality, but it also appears to make the analysis less tractable.

This paper is planned as follows. Section 2 presents the model. Section 3 contains the analysis and the key results. In Section 4, we discuss issues in empirically testing the results, as well as the implications of this research on acquiring loyal customers and enhancing brand strength. Section 5 concludes. Proofs missing from the text are given in the Appendix.

\section{The Model}

A commodity product market consists of two sellers and a unit mass of infinitesimal consumers. Consumers each have a unitary demand for the product subject to the same reservation utility $r$, and have complete information about the market prices.

We assume that seller 1 has a stronger brand name than seller 2 and that consumers differ in their brand loyalty, in the following sense. Denote seller $i$ 's price as $p_{i}, i=1,2$. A fraction $\alpha(0<\alpha<1)$ of the consumers are "switchers". A switcher is indifferent to brands and always purchases from the lower-priced seller. In the case of a price tie, she picks either seller with equal probability. The remaining $1-\alpha$ fraction of consumers are "loyal" to seller 1's product. A loyal consumer will purchase from seller 1 if $p_{1}-p_{2}<c$, where $c>0$ measures the brand strength of seller 1 relative to seller 2, will choose either brand with equal probability if $p_{1}-p_{2}=c$, and will purchase from seller 2 if $p_{1}-p_{2}>c$. Since we suppress the loyal segment size of the weak brand to zero, $1-\alpha$ may alternatively be viewed as the size difference between the loyal segments of the two brands. To emphasize the role of brand 
asymmetry in causing price promotion, we assume that the two firms have the same level of constant marginal cost, normalized to zero without loss of generality.

Here the major difference between our model and the existing ones is that we allow seller 1's brand strength, $c$, to be below the consumer reservation utility $r$. This opens the possibility that the loyal consumers of the strong brand will switch to the weak brand if the latter offers a sufficiently lower price. Since the weak brand (seller 2) does not have a loyal clientele, the asymmetric duopoly we model here may be conceived as a national brand versus a private label or store brand (as in Rao 1991), or an established brand versus a new brand.

Thus, for any $0 \leq p_{1}, p_{2} \leq r$, each seller's demand is

$$
q_{1}\left(p_{1}, p_{2}\right)= \begin{cases}1 & \text { if } p_{1}<p_{2} \\ 1-\alpha / 2 & \text { if } p_{1}=p_{2} \\ 1-\alpha & \text { if } p_{2}<p_{1}<p_{2}+c \\ (1-\alpha) / 2 & \text { if } p_{1}=p_{2}+c \\ 0 & \text { if } p_{1}>p_{2}+c\end{cases}
$$

and

$$
q_{2}\left(p_{1}, p_{2}\right)=1-q_{1}\left(p_{1}, p_{2}\right) .
$$

Here the demand specification at $p_{1}=p_{2}$ and $p_{1}=p_{2}+c$ does not affect our equilibrium, where we will see these two events occur with zero probability. Each seller's profit function is therefore

$$
w_{i}\left(p_{1}, p_{2}\right)=p_{i} q_{i}\left(p_{1}, p_{2}\right), \text { for } i=1,2 \text {. }
$$




\section{Analysis}

\subsection{Preliminaries}

First, it is easy to see that this game does not have any pure-strategy equilibrium. Apparently, the price of either firm is bounded between 0 and $r$. If $p_{1}>p_{2}+c$, then firm 1 has zero sales but can do better by deviating to $p_{2}+c$ or below. If $p_{2}<p_{1} \leq p_{2}+c$, then firm 2 will have the incentive to deviate to $p_{2}+\varepsilon<p_{1}$, where $\varepsilon>0$. If $p_{1} \leq p_{2}$, then firm 2 will benefit from deviating to $p_{1}-\varepsilon$. Since these three cases form a partition to the space of pure strategies, the desired statement follows.

Proposition 1 There is no pure strategy equilibrium in this game.

Our key task in the remainder of Section 3 is to show that there exists a unique mixed-strategy equilibrium in this game. We shall establish existence by identifying the mixed-strategy equilibrium ${ }^{7}$. Its uniqueness follows from the construction process.

Specifically, we are interested in identifying a cumulative distribution function $F_{i}^{*}\left(p_{i}\right)$ and an associated set, $P_{i}$, of prices with which firm $i$ charges with positive density, for $i=1,2$, such that

$$
\int_{P_{i}} \int_{P_{j}} w_{i}\left(p_{i}, p_{j}\right) d F_{i}^{*}\left(p_{i}\right) d F_{j}^{*}\left(p_{j}\right) \geq \int_{P_{i}} \int_{P_{j}} w_{i}\left(p_{i}, p_{j}\right) d F_{i}\left(p_{i}\right) d F_{j}^{*}\left(p_{j}\right),
$$

where $j=1,2$, and $j \neq i$.

Denote the (expected) profit function of seller $i$ when seller $j$ plays its equilibrium strategy $F_{j}^{*}\left(p_{j}\right)$ as

$$
\Pi_{i}\left(p_{i}\right)=\int_{P_{j}} w_{i}\left(p_{i}, p_{j}\right) d F_{j}^{*}\left(p_{j}\right)
$$

\footnotetext{
${ }^{7}$ An alternative approach to proving the existence of mixed-strategy equilibrium in this game is by adapting Theorem 5b of Dasgupta and Maskin (1986), which considers the case in which the only discontinuity occurs at $p_{1}=p_{2}$. However, a slight modification of the theorem to include a second point of discontinuity at $p_{1}=p_{2}+c$ is straightforward.
} 
The notion of an equilibrium requires that for $i=1$, 2 , each $p_{i} \in P_{i}$ yields the same level of expected profit, $\pi_{i}$ say, and that each $p_{i} \notin P_{i}$ yields an expected profit strictly less than $\pi_{i}$. That is, $\Pi_{i}\left(p_{i}\right)=\pi_{i}$ for $p_{i} \in P_{i}$ and $\Pi_{i}\left(p_{i}\right)<\pi_{i}$ otherwise. We observe that $\pi_{1}>0$ and $\pi_{2}>0$. To see this, note that seller 1 can always make a positive profit by selling only to its loyal consumers at price $c$. Therefore $\pi_{1}>0$, which implies that $p_{1}>0$ always. Seller 2 will also price strictly above zero, since it can attract all switchers at price $p_{1}-\varepsilon$. Hence, $\pi_{2}>0$.

Through Lemmas 1-4 below, we first establish some basic properties of the equilibrium regarding the strategy sets $P_{i}$ and the possibility of mass points in $F_{i}^{*}$.

Lemma 1 There is no mass point in $F_{2}^{*}$, and the only possible mass point in $F_{1}^{*}$ occurs at $r$.

Lemma 1 says that $F_{2}^{*}$ is continuous and that the only possible discontinuity in $F_{1}^{*}$ occurs at $r$. Because the continuity of $\Pi_{i}(p)$ is determined by the continuity of $F_{j}^{*}(p)$, by this Lemma $\Pi_{1}(p)$ is always continuous, and $\Pi_{2}(p)$ will be discontinuous at $r$ and $r-c$ if $F_{1}^{*}$ has a mass at $r$.

Lemma $2 \sup P_{2} \leq \sup P_{1}<\sup P_{2}+c$, and $\inf P_{2} \leq \inf P_{1} \leq \inf P_{2}+c$.

This Lemma is rather straightforward in light of the demand specification in our model: Any price exceeding sup $P_{2}+c$ or below inf $P_{2}$ is a dominated strategy for seller 1 , and any price exceeding $\sup P_{1}$ or below inf $P_{1}-c$ is a dominated strategy for seller 2.

Lemmas 1 and 2 jointly lead to the next Proposition.

Proposition 2 Any equilibrium in this game is one of the following three types: (1) Type-I: $\sup P_{1}=\sup P_{2}=r$, with $F_{1}^{*}$ having a mass point at 
r; (2)Type-II: $\sup P_{2}<\sup P_{1}=r$ with $F_{1}^{*}$ having a mass at $r$; and (3) Type-III: $\sup P_{2}<\sup P_{1}$, with neither firm's strategy having a mass point.

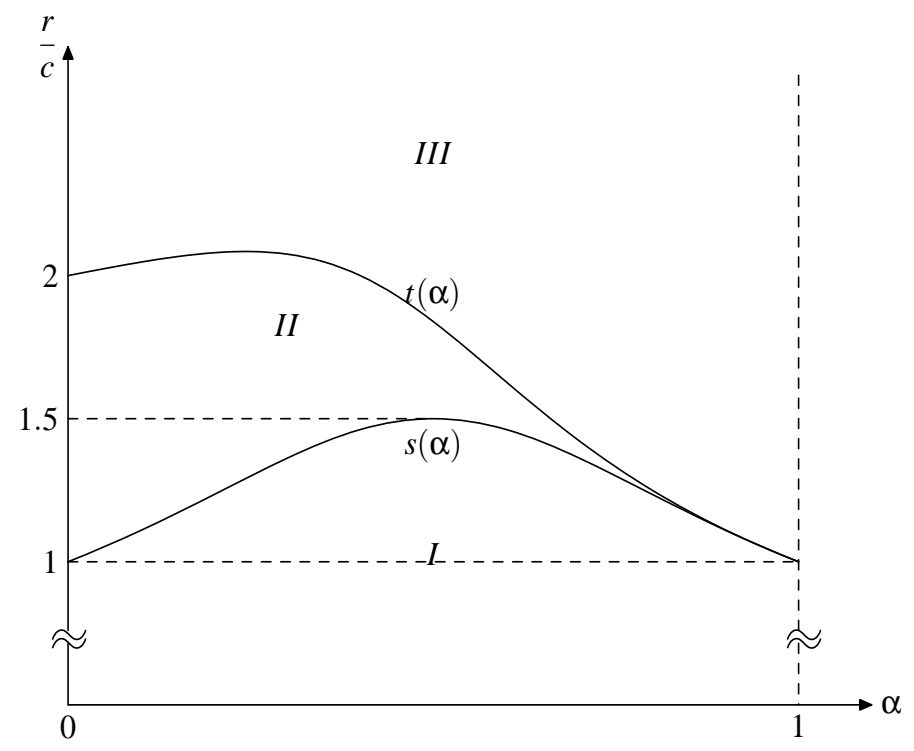

Figure 1

Lemma 3 For any $p$, we can find $\varepsilon>0$ such that if $(p, p+\varepsilon) \subset P_{1}$, then either $(p, p+\varepsilon) \subset P_{2}$ or $(p-c, p-c+\varepsilon) \subset P_{2}$. For any $p$, we can find $\varepsilon>0$ such that if $(p, p+\varepsilon) \subset P_{2}$, then either $(p, p+\varepsilon) \subset P_{1}$ or $(p+c, p+c+\varepsilon) \subset P_{1}$.

Lemma 3 can be readily shown by contradiction. Suppose $(p, p+\varepsilon) \subset P_{1}$, $(p, p+\varepsilon) \cap P_{2}=\phi$ and $(p-c, p-c+\varepsilon) \cap P_{2}=\phi$. Then $\Pi_{1}$ would be increasing over $(p, p+\varepsilon)$, contradicting the equal-profit condition for $(p, p+\varepsilon) \subset P_{1}$. From this, the first statement of the Lemma follows. The argument for the second statement is analogous. The next lemma shows that the spread of each strategy set $P_{i}$ is bounded by $2 c$.

Lemma $4 \sup P_{i}-\inf P_{i}<2 c$. 
In the technical Appendix, this Lemma is used to establish an even tighter bound on each firm's price range $\left(\sup P_{i}-\inf P_{i}<c\right)$.

\subsection{The Price Equilibrium}

Proposition 2 above has identified all three possible types of equilibria. Which type will realize turns out to depend on the parameter values along two dimensions, $r / c$ and $\alpha$ (see Figure 1). Here $\alpha$ is the population of switchers and $r / c$ is a measure of seller 1's brand strength relative to consumer reservation utility-the weaker the brand strength, the higher this ratio is. Type I (II, III) equilibrium obtains in Region I (II, III, respectively) of Figure 1. We next describe each type of equilibrium and discuss its key properties.

Proposition 3 (Type-I Equilibrium) When $r / c \leq s(\alpha) \equiv(1-\alpha+$ $\left.\alpha^{2}\right) /\left(1-2 \alpha+2 \alpha^{2}\right)$, there is a unique mixed-strategy equilibrium

$$
\begin{aligned}
& F_{1}^{*}(p)= \begin{cases}1-\frac{r(1-\alpha)}{p} & \text { for } r(1-\alpha) \leq p<r \\
1 & \text { for } p \geq r\end{cases} \\
& F_{2}^{*}(p)=\frac{1}{\alpha}\left[1-\frac{r(1-\alpha)}{p}\right] \quad \text { for } r(1-\alpha) \leq p<r .
\end{aligned}
$$

In this equilibrium, $\sup P_{1}=\sup P_{2}=r$, and $F_{1}^{*}$ has a mass of $1-\alpha$ at $r$.

Type-I equilibrium obtains when the magnitude of seller 1's brand equity is sufficiently high $(c \geq r / s(\alpha))$. It has the following key features: (1) Both sellers price in the same range, and thus have the same regular price (at or arbitrarily close to $r$ ) and the same lowest price. (2) The strategy of the strong brand has a mass $1-\alpha$ at $r$. Thus the strong brand promotes less frequently. (3) Since $F_{1}^{*}$ (first-degree) stochastically dominates $F_{2}^{*}, E p_{1}>$ $E p_{2}$, i.e., on average the strong brand enjoys a price premium over its rival. (4) $E\left[p_{1} \mid p_{1}<r\right]=E\left[p_{2} \mid p_{2}<r\right]$, i.e., the average depth of discount is the same for both sellers. Type-I equilibrium therefore has the same set of essential spirits as the one in Narasimhan (1988) developed under the assumption 
that a loyal customer is captive to her favored brand. This resemblance is not surprising after some closer examination of the condition in Proposition 3: $r / c \leq s(\alpha)$ implies $r / c \leq 1 / \alpha$, under which $\sup P_{1}-\inf P_{2}=\alpha r \leq c$. Therefore, in Type-I equilibrium the loyal consumers of seller 1 are de facto "captive," in the sense that they will never purchase from seller 2. Although seller 2 can potentially attract seller 1's loyal consumers at a positive price, it is to seller 2's best interest just to sell to the switchers.

From Proposition 3, we can readily derive the following comparative statics. The expected profits are $\pi_{1}=r(1-\alpha)$ and $\pi_{2}=r(1-\alpha) \alpha$. Seller 1 's profit is what it would earn by serving only its loyal consumers at a price equal to their reservation utility. The expected prices are $E p_{1}=$ $r(1-\alpha)(1-\ln (1-\alpha))$ and $E p_{2}=-r(1-\alpha) / \alpha \ln (1-\alpha)$. Since in this type of equilibrium the loyal consumers always buy from seller 1, $E p_{1}$ is also the mean price they face. The switchers always buy at the lower price, which has a distribution function $G(p)=1-\left(1-F_{1}^{*}(p)\right)\left(1-F_{2}^{*}(p)\right)$, and face an expected price $r\left[2(1-\alpha)+(1-\alpha)^{2} / \alpha \ln (1-\alpha)\right]$.

In Type-I equilibrium, as a increases from zero to 1 , price competition intensifies because a larger segment of switchers becomes more appealing to both sellers. The following holds as $\alpha$ increases: (1) both $E p_{1}$ and $E p_{2}$ decrease, (2) the lower bound of the common price range decreases, and (3) $\pi_{2}$ increases when $\alpha \leq 0.5$ and decreases when $\alpha>0.5$, while $\pi_{1}$ decreases. Not surprisingly, a larger switcher segment hurts the profit of the strong brand. Since in Type-I equilibrium seller 2 sells only to the switchers, its sales are diminished when $\alpha$ is small. When $\alpha$ approaches 1 , price competition becomes very fierce $\left(E p_{1} \longrightarrow 0\right.$ and $\left.E p_{2} \longrightarrow 0\right)$. This explains the unimodality of $\pi_{2}$.

Proposition 4 (Type-II Equilibrium) When $s(\alpha)<r / c<t(\alpha)$, there is 
a unique mixed-strategy equilibrium ${ }^{8}$

$$
\begin{aligned}
& F_{1}^{*}(p)=\left\{\begin{array}{ll}
1-\frac{\pi_{2}}{\alpha p} & \text { for } \frac{\pi_{2}}{\alpha} \leq p<\frac{\pi_{1}}{1-\alpha} \\
\frac{1}{1-\alpha}\left[1-\frac{\pi_{2}}{p-c}\right] & \text { for } \frac{\pi_{1}}{1-\alpha} \leq p<r \\
1 & \text { for } p \geq r
\end{array},\right. \\
& F_{2}^{*}(p)=\left\{\begin{array}{lll}
1-\frac{\pi_{1}}{(1-\alpha)(p+c)} & \text { for } \frac{\pi_{1}}{1-\alpha}-c \leq p<r-c \\
1-\frac{\pi_{1}}{r(1-\alpha)} & \text { for } r-c \leq p<\frac{\pi_{2}}{\alpha} \\
\frac{1}{\alpha}\left[1-\frac{\pi_{1}}{p}\right] & \text { for } \frac{\pi_{2}}{\alpha} \leq p<\frac{\pi_{1}}{1-\alpha} \\
1 & \text { for } p \geq \frac{\pi_{1}}{1-\alpha}
\end{array}\right.
\end{aligned}
$$

where

$$
\begin{aligned}
& \pi_{1}=\frac{c(1-\alpha)}{2 \alpha^{2}}\left(\alpha^{2}-\theta(1-\alpha)^{2}+\sqrt{\left(\alpha^{2}-\theta(1-\alpha)^{2}\right)^{2}+4 \alpha^{2} \theta(1-\alpha)}\right), \\
& \pi_{2}=\frac{1}{\frac{1-\alpha}{\alpha \pi_{1}}+\frac{1}{r(1-\alpha)}}, \text { and } \theta \equiv \frac{r}{c}
\end{aligned}
$$

In this equilibrium, seller 1's strategy has a mass at $\sup P_{1}=r$.

Type-II equilibrium obtains for intermediate values of brand loyalty (i.e., $r / t(\alpha)<c<r / s(\alpha))$, and $F_{1}^{*}$ and $F_{2}^{*}$ are graphed in Figure 2. In Type-II equilibrium, seller 1's strategy has a mass of $\left(\pi_{2}-\alpha(r-c)\right) /[(1-\alpha)(r-c)]$ at $r$, meaning that the strong brand still promotes less frequently than the weak brand, just as in Type-I equilibrium. From Proposition 4, we obtain the following distinguishing properties of Type-II equilibrium.

Proposition 5 In Type-II equilibrium, (1) $\sup P_{2}<\sup P_{1}=r$, inf $P_{2}<$ $\inf P_{1}, \sup P_{1}-\inf P_{1}<\sup P_{2}-\inf P_{2}=c$; (2) $P_{2}$ is nonconvex.

\footnotetext{
${ }^{8} t(\alpha)=\frac{1}{3\left(1-3 \alpha+3 \alpha^{2}\right)}\left(7 \alpha^{2}-7 \alpha+2-\frac{2^{\frac{1}{3}} h}{k}+\frac{k}{2^{\frac{1}{3}}}\right)$, where $h=-4+19 \alpha-35 \alpha^{2}+26 \alpha^{3}-4 \alpha^{4}$, $k=\left(16-114 \alpha+342 \alpha^{2}-521 \alpha^{3}+387 \alpha^{4}-87 \alpha^{5}-16 \alpha^{6}+\sqrt{3} \alpha(3(1-3 \alpha+\right.$ $\left.\left.\left.3 \alpha^{2}\right)\right) \sqrt{-4+36 \alpha-112 \alpha^{2}+168 \alpha^{3}-117 \alpha^{4}+32 \alpha^{5}}\right)^{\frac{1}{3}}$
} 


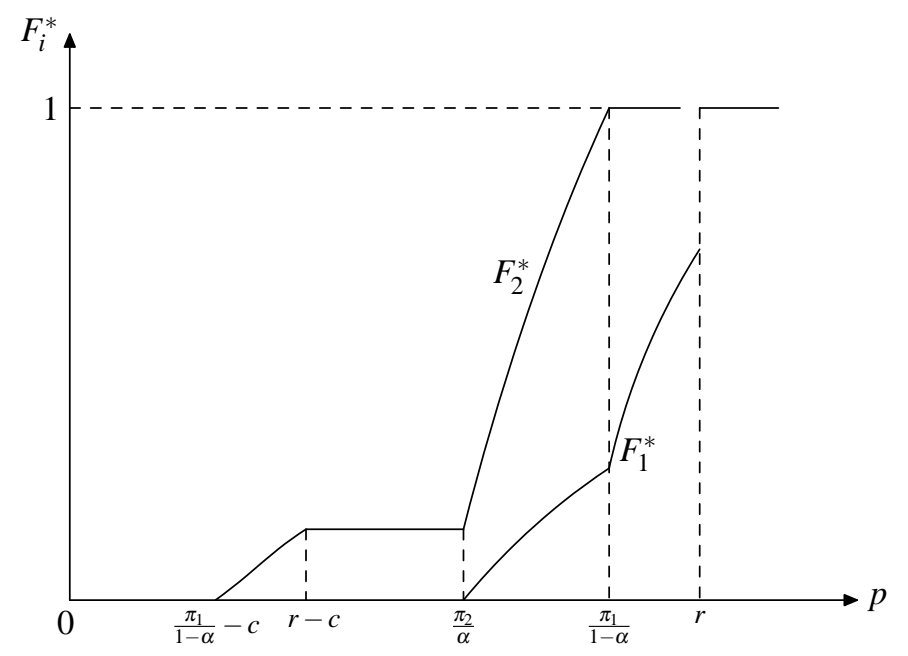

Figure 2

Part (1) of the Proposition implies that $\sup P_{1}-\inf P_{2}>c$. Therefore, the strong brand's loyal consumers are no longer captive and will purchase from the weak brand with positive probability. Following the convention in the literature, we interpret $\sup P_{i}$ as firm $i$ 's regular price. Then the strong brand has a higher regular price than the weak brand. Seller 2 has a nonconvex strategy set and will not charge any price in $\left[r-c, \pi_{2} / \alpha\right]$. Despite $\sup P_{1}-\inf P_{1}<\sup P_{2}-\inf P_{2}$, we can verify that the price ranges of the two sellers have the same effective length $r-\pi_{2} / \alpha$, due to the gap in $P_{2}$.

We now compare the mean promotional depth of the two sellers. We measure seller $i$ 's promotional depth as the difference between its regular price and the mean of its price below its regular price, i.e., $\sup P_{i}-E\left[p_{i} \mid p_{i}<\right.$ $\left.\sup P_{i}\right]$. We then have

$$
\sup P_{1}-E\left[p_{1} \mid p_{1}<\sup P_{1}\right]=r-\frac{E p_{1}-r\left(1-\operatorname{Pr}\left(p_{1}<r\right)\right)}{\operatorname{Pr}\left(p_{1}<r\right)}
$$

and

$$
\sup P_{2}-E\left[p_{2} \mid p_{2}<\sup P_{2}\right]=\sup P_{2}-E p_{2},
$$


where the second equality is due to the lack of mass point in seller 2's strategy. The exact expressions of $E p_{1}$ and $E p_{2}$ are given in the Appendix. Through numerical methods, we obtain the next Proposition.

Proposition 6 In Type-II equilibrium, seller 1 has a larger (smaller) average promotional depth than seller 2 when $\alpha$ is relatively small (large).

The intuition behind Proposition 6 is as follows. When the switcher population is relatively small, seller 2 , the weak brand, has a strong incentive to invade seller 1's loyal segment by offering discounts deeper than $c$ (see the discussion after Proposition 7 below). Since seller 1 has a higher regular price than seller $2\left(\sup P_{1}=r>\sup P_{2}\right)$, it is forced to offer discounts that on average exceeds seller 2's in order to defend its loyal customer base. On the other hand, when the switcher population is large, seller 2's regular price $\left(\sup P_{2}=\pi_{1} /(1-\alpha)\right)$ become closer to seller 1's regular price $(r)$, but seller 1 has a strong incentive to attract the switchers. This forces seller 2 to offer a higher average discount to prevent losing too many switchers to its rival. Figure 3 depicts the average promotional depth of the two sellers for $r=1.55$, $c=1$, and $0<\alpha<0.636$. The same pattern of relative promotional depth between the two sellers holds for other parameter configurations of Type-II Equilibrium.

Proposition 6 compares the promotional depth of the two brands in TypeII equilibrium, where seller 1's brand equity is not very strong relative to seller 2's. This Proposition directly contrasts with our Type-I equilibrium or Narasimhan (1988), where the two brands have the same average promotional depth. Proposition 12 of Raju et al (1990) also addresses the case in which neither brand is very strong relative to the other so that each brand actively attracts consumers loyal to the other, and finds that it is the weaker brand that offers a larger discount. The difference between their conclusion and ours is mainly due to their assumption of equally-sized loyal segments. We can verify that when $\alpha=0.5$ in our model, the weak brand also offers a higher average discount. 


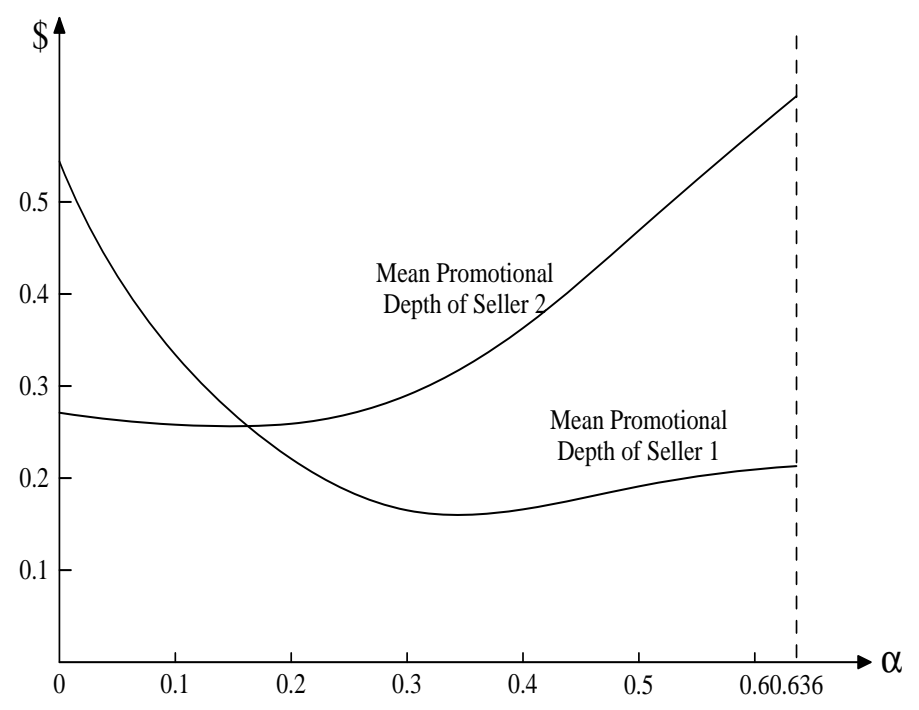

Figure 3

The two sellers' price ranges overlap in $\left[\pi_{2} / \alpha, \pi_{1} /(1-\alpha)\right)$. We can verify that $E\left[p_{1} \mid \pi_{2} / \alpha \leq p_{1}<\pi_{1} /(1-\alpha)\right]=E\left[p_{2} \mid \pi_{2} / \alpha \leq p_{2}<\pi_{1} /(1-\alpha)\right]$, i.e., they charge the same mean price in their overlapping price region. The two sellers' expected profits are given in Proposition 4. We can readily compute the expected prices of the sellers $\left(E p_{1}\right.$ and $\left.E p_{2}\right)$ and the expected prices paid by a loyal consumer and a switcher $\left(E p^{l}\right.$ and $\left.E p^{s}\right)$. The closed-form expressions for these metrics are rather complex and thus relegated to the Appendix. In Figure 4, we plot these metrics as functions of $\alpha$ for $r=1.55$, $c=1$, and $0<\alpha<0.636$. The next Proposition summarizes some related key comparative statics.

Proposition 7 In Type-II equilibrium, as $\alpha$ increases from zero, (1) the mass of seller 1's strategy at $r,\left(\pi_{2}-\alpha(r-c)\right) /[(1-\alpha)(r-c)]$, first increases and then decreases; (2) both $E p_{1}$ and $E p_{2}$ first increase and then decrease.

Recall that in Type-I equilibrium, the mass of $F_{1}^{*}$ at $r, E p_{1}$ and $E p_{2}$ are all monotonically decreasing in $\alpha$. In contrast, they are all unimodal func- 


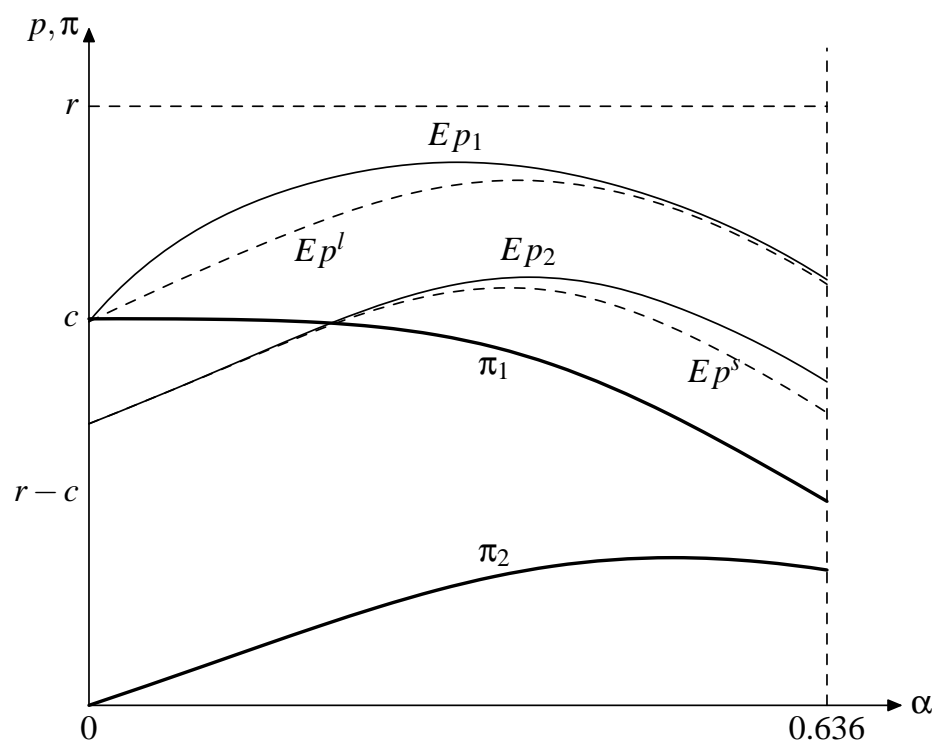

Figure 4

tions of $\alpha$ in Type-II equilibrium. Part (1) of Proposition 7 indicates that extreme values of switcher population weaken the strong brand's ability to maintain its price at the regular level. $E p_{1}$ and $E p_{2}$ are graphed in Figure 4. In Type-II equilibrium, when $\alpha$ gets smaller, seller 2 will be less content with selling only to the thin slice of switchers, and more inclined to attract seller 1 's loyal consumers by offering discounts deeper than $c$. To better defend its loyal segment, seller 1 is forced to lower its price correspondingly. On the other hand, when $\alpha$ gets larger, even seller 1 itself has a stronger incentive to compete for a fat share of switchers, again heating up competition. Therefore, in Type-II equilibrium, price competition is most intensified for extreme values of $\alpha$, and is most relaxed for intermediate values of $\alpha$. This explains the unimodality of $E p_{1}, E p_{2}$ and the mass in $F_{1}^{*}$.

Lastly, $\pi_{1}$ is monotonically decreasing in $\alpha$ and $\pi_{2}$ is unimodal, just as in Type-I equilibrium. Unlike Type-I equilibrium, however, here $\pi_{1}(\alpha)$ is nonlinear (see Figure 4).

Proposition 8 (Type-III Equilibrium) When $r / c \geq t(\alpha)$, there is a 
unique mixed-strategy equilibrium:

$$
\begin{aligned}
& F_{1}^{*}(p)=\left\{\begin{array}{ll}
1-\frac{\pi_{2}}{\alpha p} & \text { for } \frac{\pi_{2}}{\alpha} \leq p<\frac{\pi_{1}}{1-\alpha} \\
\frac{1}{1-\alpha}\left[1-\frac{\pi_{2}}{p-c}\right] & \text { for } \frac{\pi_{1}}{1-\alpha} \leq p<\frac{\pi_{2}}{\alpha}+c
\end{array},\right. \\
& F_{2}^{*}(p)= \begin{cases}1-\frac{\pi_{1}}{(1-\alpha)(p+c)} & \text { for } \frac{\pi_{1}}{1-\alpha}-c \leq p<\frac{\pi_{2}}{\alpha} \\
\frac{1}{\alpha}\left[1-\frac{\pi_{1}}{p}\right] & \text { for } \frac{\pi_{2}}{\alpha} \leq p<\frac{\pi_{1}}{1-\alpha}\end{cases}
\end{aligned}
$$

where

$$
\pi_{1}=\frac{c(1-\alpha)^{2} t(\alpha)[t(\alpha)-1]}{\alpha+(1-2 \alpha) t(\alpha)}, \text { and } \pi_{2}=c \alpha(t(\alpha)-1) .
$$

In this equilibrium, $\sup P_{2}<\sup P_{1}<r$ and neither firm's strategy has a mass point.

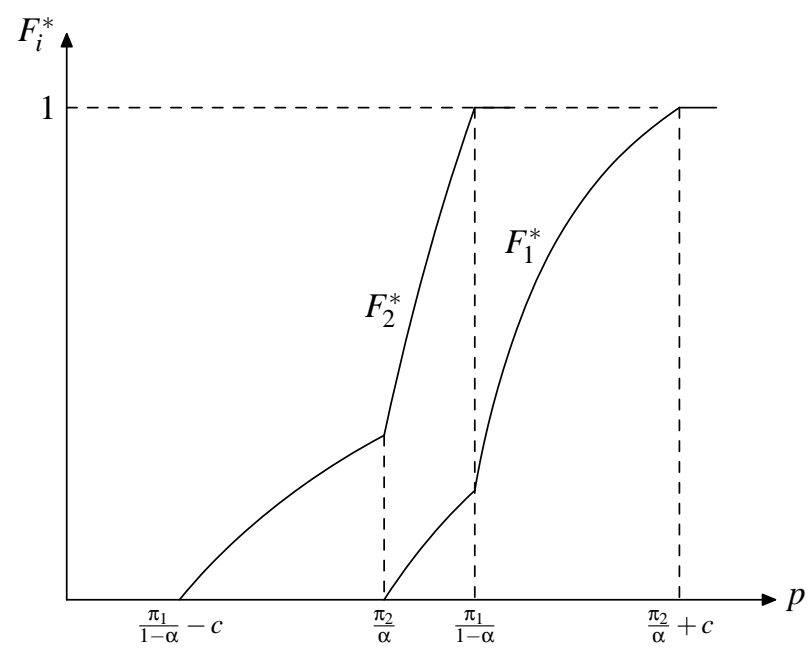

Figure 5

The equilibrium distribution functions are graphed in Figure 5. When seller 1 has a relatively low brand strength (i.e., $c \leq r / t(\alpha)$ ), Type-III equilibrium obtains, where competition is more intense than the other two types of equilibria so that even the strong brand can not price up to consumer reservation utility and that neither firm's strategy contains a mass point. 
Both brands promote equally frequently in Type-III equilibrium. Compared with Type-II equilibrium, $P_{2}$ is now convex.

Otherwise, Type-III equilibrium shares many key features as Type-II equilibrium. First, seller 1's loyal consumers are not captive, as sup $P_{1}-$ $\inf P_{2}>c$. Second, seller 1 has a higher price range and a higher regular price than seller $2, \operatorname{as} \sup P_{2}<\sup P_{1}$ and $\sup P_{i}-\inf P_{i}=c, i=1,2$. Third, both $E p_{1}$ and $E p_{2}$ are unimodal functions of $\alpha$ (cf. Proposition 7, part (2)). We can also verify that $E\left[p_{1} \mid \pi_{2} / \alpha \leq p_{1}<\pi_{1} /(1-\alpha)\right]=E\left[p_{2} \mid \pi_{2} / \alpha \leq p_{2}<\right.$ $\left.\pi_{1} /(1-\alpha)\right]$. That is, the two sellers' mean prices in their overlapping price region are identical. The rationale underlying these properties also resembles that in Equilibrium II. Lastly, the mean promotional depth of the strong brand can be greater or smaller than that of the weak brand. However, unlike Type-II equilibrium, here the critical value of $\alpha$ does not depend on either $r$ or $c$, as shown in the next Proposition.

Proposition 9 In Type-III equilibrium, seller 1 has a larger (smaller) average promotional depth than seller 2 when $\alpha<\alpha^{*} \simeq 0.442$ (when $\alpha>\alpha^{*}$ ).

The key comparative statics of Equilibrium III are relegated to the Appendix. The price ranges and expected profits of the two sellers are plotted in Figure 6. The grey area on the top (bottom) is seller 1's (seller 2's) exclusive price range, and the dark grey area in the middle is the two sellers' overlapping price range. As $\alpha$ decreases, we see greater dispersion in the prices of the two sellers because seller 2 becomes more aggressive in attracting seller 1 's loyal consumers by undercutting its price by more than $c$.

\section{Discussions and Implications}

In this Section we attempt to provide a synthesis of all three types of equilibria. We first examine how varying the values of $\alpha$ and $c$, respectively, will impact each firm's profits, and then discuss issues in empirically testing some of the key results. 


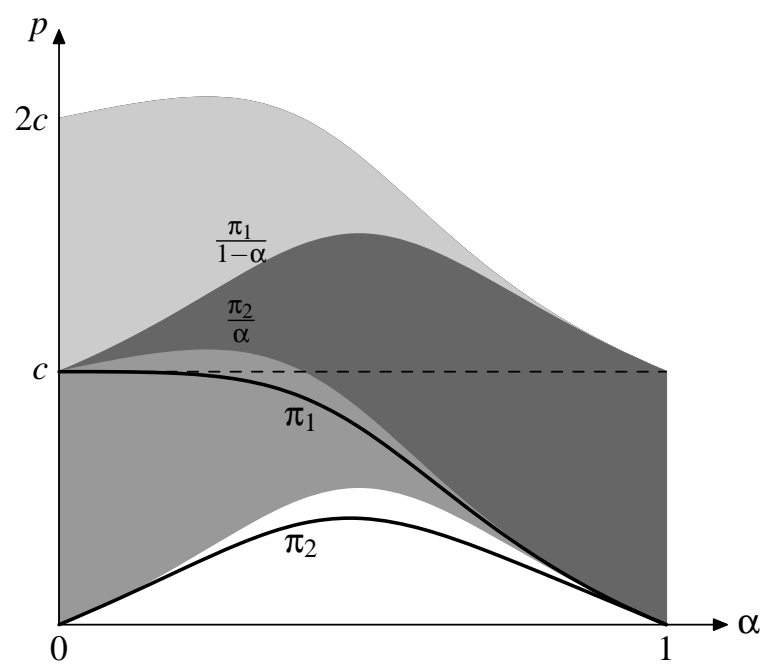

Figure 6

\subsection{Implications on Acquiring Loyal Customers}

In reality, a firm may be able to convert some brand switchers into its loyal customers, e.g., through persuasive advertising. We now turn our attention to examining seller 1's return from such customer acquisition efforts. In all three types of equilibria, $\pi_{1}$ and $\pi_{2}$ are decreasing and unimodal functions of $\alpha$, respectively. Thus the strong brand always benefits from a larger loyal customer base, but the weak brand does not always benefit from a larger switcher population. However, seller 1 enjoys different rates of return from customer acquisition in these three types of equilibria. In Type-I equilibrium, $\pi_{1}$ increases linearly as $\alpha$ decreases, i.e., it receives constant marginal return from converting switchers into its loyal customers. In the remaining two types of equilibria, its returns in customer acquisition are nonlinear. In particular, for small values of $\alpha$, seller 1's profit improves slowly as $\alpha$ further decreases (see Figures 4 and 6). Therefore, when $r / c>1$, seller 1's customeracquisition effort may yield diminished return once its loyal customer base becomes sufficiently large.

For example, suppose $r=1.55$ and $c=1$. When $\alpha \leq 0.636$, Type-II 
equilibrium obtains. We can verify that, at $\alpha=0.30$, seller 1 already makes an expected profit of 0.958 . Even if seller 1 could transform all the remaining switchers into its loyal customers, its expected profit can only increase by 0.042 or $4.4 \%$.

The fact that $\pi_{2}$ is unimodal in $\alpha$ suggests that when $\alpha$ is relatively high, the weak brand will benefit from the strong brand's effort to convert the brand switchers into its own loyal customers. As was pointed out earlier, here the reason is that a large proportion of switchers in the market intensifies price competition and thus hurts both sellers' profits. In such a case, efforts by the strong brand in reducing $\alpha$ may benefit both brands.

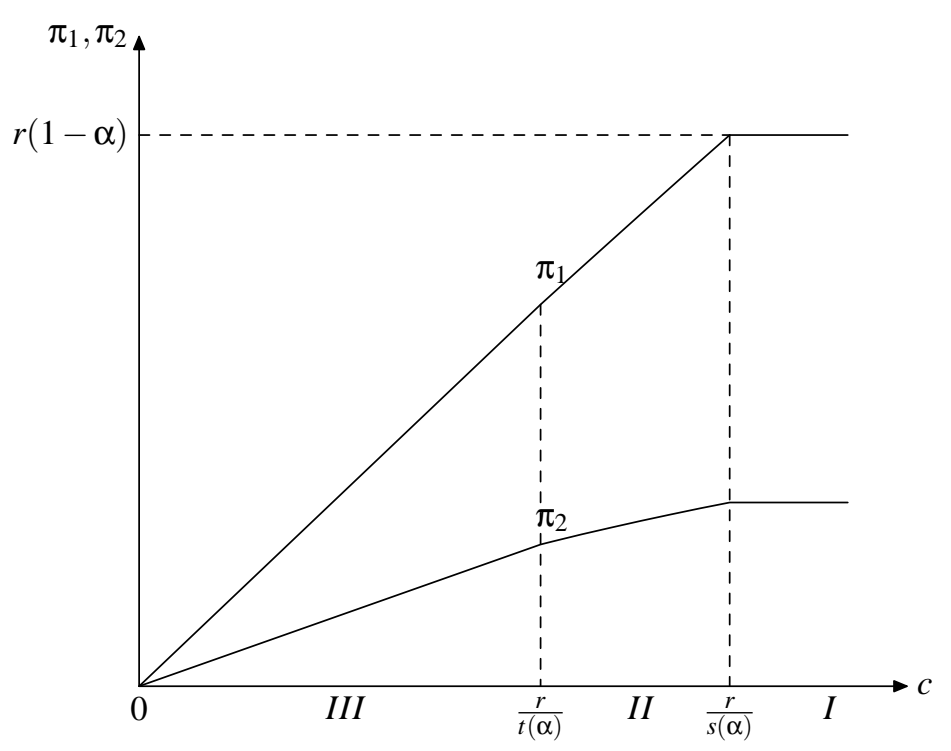

Figure 7

\subsection{Implications on Enhancing Brand Strength}

It is often believed that advertising can lead to product differentiation and reduce consumers' price sensitivity (e.g., Comanor and Wilson 1974, Mitra and Lynch 1995, Kaul and Wittink 1995, Mela et al 1997 and Shum 2004). 
Over time a firm may be able to increase its brand equity through non-priceoriented advertising and brand building. We now discuss the implications of raising seller 1's brand advantage $c$ on both sellers' profits. For any given $\alpha$ and $r$, as $c$ increases the equilibrium goes through Types III, II, and I. The two sellers' expected profits are shown as functions of $c$ in Figure 7. Raising seller 1's brand strength will increase both sellers' profits linearly in Type III equilibrium and almost linearly in Type-II equilibrium. Therefore, brand enhancement by seller 1 has positive externalities, as it also leads to a higher expected profit for seller 2. That a stronger brand of seller 1 benefits its competitor seems somewhat puzzling at first. The reason is that seller 1's brand strength $c$ essentially measures the degree of product differentiation perceived by its loyal consumers, and a higher $c$ serves to soften price competition and thus benefits both sellers. In this sense enhancing seller 1's brand strength relaxes price competition in a manner reminiscent of raising the product quality of the high-end firm in the vertically differentiated duopoly of Shaked and Sutton (1982) and Moorthy (1988). For example, when $c=0$, Bertrand competition prevails and both sellers price at their marginal costs (normalized to zero).

\subsection{Empirical Analysis}

One naturally wonders to what extent the results produced with our stylized model can be replicated in reality. To empirically test our results, we can offer the following considerations.

1. When the brand equity of the strong brand is moderate or low relative to consumer reservation utility (as in Types II and III equilibria), our model predicts that the strong brand enjoys a higher price range and a higher regular price than the weak brand. Such a prediction appears to be supported by casual observations in many product markets such as packaged goods and grocery items, among others.

2. When the strong brand has a moderate or high brand equity (as in 
Types II and I equilibria), its strategy has a mass point while the weak brand's strategy does not, meaning that the former promotes less frequently than the latter, consistent with Narasimhan (1988) and Raju et al. (1990). In contrast, neither firm's strategy has a mass point when the strong brand has a relatively low brand equity (as in Type-III equilibrium). Therefore, when the strong brand can not command a sufficiently high price premium from its loyal consumers, it will be in a less favorable position to maintain its regular price. Instead, it may be forced to offer discounts as frequently as its competitor. This implies that in markets where no seller has a sufficiently superior brand advantage, one is less likely to observe significant differences in promotional frequency among the sellers. For example, this may be true when consumers can examine a product and determine its quality with ease, as brand names tend to be less important in such markets (Png and Reitman 1995).

Existing studies have produced different perspectives on the relative promotional frequency between two asymmetric brands. Rao (1991) predicts that the weak brand promotes less often than the strong brand, opposite to Narasimhan (1988) and Raju et al. (1990). Therefore, the contrast between their results may be reconciled, to a certain extent, by our result that the two brands promote equally frequently when the strong brand possesses only mild relative strength.

3. In Type-I equilibrium, the average promotional depths by the two firms are equal, as in Narasimhan (1988). In the other two types of equilibria, however, the promotional depth of one brand may be higher or lower than the other, depending on the population of switchers. Blattberg and Wisniewski (1989) find empirical evidence that in part supports our prediction: the mean deal depth for national brands of tuna and flour exceeds that for private labels. 


\section{Conclusion}

In the Narasimhan (1988) model consisting of a strong brand (seller 1) and a weak brand (seller 2), this article has succeeded in relaxing a major assumption employed in most previous studies on price promotion-namely, consumers loyal to a brand are entirely captive to that particular brand. We allow the relative strength of the strong brand to fall below consumer reservation utility. In this setting, a unique mixed-strategy price equilibrium exists and falls into one of three possible types depending on the parameter configuration of consumer reservation utility, brand strength, and the switcher population. The type of equilibrium when seller 1's brand equity is relatively high corresponds to the original equilibrium obtained by Narasimhan (1988). In this type of equilibrium, the loyal consumers are de facto captive. When seller 1's brand equity is moderate and low, respectively, two new types of equilibria emerge in which seller 1's loyal consumers purchase from seller 2 with positive probability. As shown above, these two types of equilibria demonstrate very distinctive patterns than the first type. Therefore, it is our hope that this analysis has provided a more complete picture about firms' pricing and consumers' choice behavior under finite brand loyalty.

We note that allowing finite brand loyalty has significantly complicated our analysis, because each firm's demand function is now discontinuous at two points (when $p_{1}=p_{2}$ and when $p_{1}=p_{2}+c$ ). In contrast, under the usual assumption that the loyal consumers never switch, each firm's demand function is discontinuous only when $p_{1}=p_{2}$. Lastly, our current model has only considered two competing firms with different sizes of loyal clientele. It may be potentially fruitful to carry out the current analysis in a symmetric duopoly or oligopoly. In particular, analysis of an oligopoly can help shed additional light on how the number of players may impact each firm's promotional decisions in the presence of finite brand loyalty. 


\section{Appendix}

\section{Proof of Lemma 1.}

Proof: First, we show that seller 2's strategy has no mass point. Suppose instead that seller 2's strategy has a mass $w$ at $p$, then charging $p$ gives seller 1 an expected profit

$$
\begin{aligned}
\Pi_{1}(p)= & p\left(1-F_{2}^{*}(p)\right)+(1-\alpha) p\left(F_{2}^{*}(p)-F_{2}^{*}(p-c)-w\right) \\
& +(1-\alpha / 2) p w+p(1-\alpha) / 2 \operatorname{Pr}\left(p_{2}=p-c\right),
\end{aligned}
$$

and charging $p+\epsilon$ gives seller 1 an expected profit

$$
\begin{aligned}
\Pi_{1}(p+\epsilon)= & (p+\epsilon)\left(1-F_{2}^{*}(p+\epsilon)\right)+(1-\alpha)(p+\epsilon)\left(F_{2}^{*}(p+\epsilon)-F_{2}^{*}(p+\epsilon-c)\right) \\
& +(1-\alpha / 2)(p+\epsilon) \operatorname{Pr}\left(p_{2}=p+\epsilon\right)+(p+\epsilon)(1-\alpha) / 2 \operatorname{Pr}\left(p_{2}=p+\epsilon-c\right) .
\end{aligned}
$$

Since $F_{2}^{*}(p)$ is a right continuous function, we have $F_{2}^{*}(p+\epsilon) \rightarrow F_{2}^{*}(p)$, $F_{2}^{*}(p+\epsilon-c) \rightarrow F_{2}^{*}(p-c), \operatorname{Pr}\left(p_{2}=p+\epsilon\right) \rightarrow 0$ and $\operatorname{Pr}\left(p_{2}=p+\epsilon-c\right) \rightarrow 0$ as $\epsilon \rightarrow 0$. Thus, $\lim _{\epsilon \rightarrow 0} \Pi_{1}(p)-\Pi_{1}(p+\epsilon)=\alpha w p / 2+p(1-\alpha) / 2 \operatorname{Pr}\left(p_{2}=\right.$ $p-c) \geq \alpha w p / 2>0$.

Similarly, we can show that $\lim _{\epsilon \rightarrow 0} \Pi_{1}(p-\epsilon)-\Pi_{1}(p)>0$.

If $p<r$, the above analysis suggests that $\exists \delta>0$ such that $[p, p+\delta) \cap P_{1}=$ $\varnothing$.

If $p+c<r$, by the same method we can show that

$$
\begin{aligned}
\lim _{\epsilon \rightarrow 0} \Pi_{1}(p+c)-\Pi_{1}(p+c+\epsilon) & =(p+c) \alpha / 2 \operatorname{Pr}\left(p_{2}=p+c\right)+(p+c)(1-\alpha) / 2 w \\
& \geq(p+c)(1-\alpha) / 2 w>0
\end{aligned}
$$

and that $\lim _{\epsilon \rightarrow 0} \Pi_{1}(p+c-\epsilon)-\Pi_{1}(p+c)>0$. This indicates that $\exists \delta>0$ such that $[p+c, p+c+\delta) \cap P_{1}=\varnothing$.

When $p<r$, the above implies that seller 2 can always increase its profit by moving its mass at $p$ to $p+\delta_{1}$, where $0<\delta_{1}<\delta$, a contradiction to the 
assumption that seller 2's strategy has a mass at $p$.

When $p=r, \sup P_{1}=r \operatorname{since} \sup P_{1} \geq \sup P_{2}$. If $F_{1}^{*}$ has a mass at $r$, it's easy to show that $\lim _{\epsilon \rightarrow 0} \Pi_{2}(p-\epsilon)>\Pi_{2}(p)$. If $F_{1}^{*}$ doesn't have a mass at $r, \Pi_{2}(r)=0$. Therefore we can't have $r \in P_{2}$. We have show that seller 2 's equilibrium strategy doesn't contain a mass point.

Analogously, if seller 1's equilibrium strategy has a mass at $p<r$, then we can show that $\exists \delta>0$ such that $[p, p+\delta) \cap P_{2}=\varnothing$ and $[p-c, p-c+\delta) \cap P_{2}=\varnothing$, which implies that seller 1 can increase its profit by moving its mass at $p$ to slightly above $p$, a contradiction. Thus, seller 1's equilibrium strategy can't have a mass at $p<r$. Notice, however, it's still possible for seller 1's strategy to have a mass at $r$. Q.E.D.

\section{Proof of Lemma 2.}

Proof: Any price $p_{1}>\sup P_{2}+c$ yields zero profit for seller 1 , and any price $p_{2}>\sup P_{1}$ yields zero profit for seller 2 . Hence, $\sup P_{2} \leq \sup P_{1} \leq$ $\sup P_{2}+c$. Suppose $\sup P_{1}=\sup P_{2}+c$ in equilibrium. Recall, Lemma 1 states that $F_{2}^{*}$ has no mass point, and that the only possible mass point of $F_{1}^{*}$ is at $r$. Then at a price $p$ immediately below $\sup P_{1}$, firm 1 obtains a profit $\Pi_{1}(p)=(1-\alpha) p\left[1-F_{2}^{*}(p-c)\right]$. As $p \longrightarrow \sup P_{1}, \Pi_{1}(p) \longrightarrow 0$. This contradicts the continuity of $\Pi_{1}(p)$ if $\sup P_{1}-\varepsilon \notin P_{1}$ for an arbitrarily small $\varepsilon$, and contradicts $\pi_{1}>0$ otherwise.

For seller 1 , a price $p_{1}<\inf P_{2}$ is strictly dominated by $p_{1}+\varepsilon<\inf P_{2}$, which implies $\inf P_{2} \leq \inf P_{1}$. Likewise, for seller 2 , a price $p_{2}<\inf P_{1}-c$ is strictly dominated by $p_{2}+\varepsilon<\inf P_{1}-c$. This implies inf $P_{1} \leq \inf P_{2}+c$. Q.E.D.

\section{Proof of Proposition 2.}

Proof: From Lemma 2, we have either $\sup P_{2}=\sup P_{1}$ or $\sup P_{2}<\sup P_{1}$. We first show that if $\sup P_{1}=\sup P_{2}$, then $\sup P_{1}=\sup P_{2}=r$ and $F_{1}^{*}$ has a mass at $r$. Suppose $\sup P_{1}=\sup P_{2}$. Then for a price $p \in P_{2}$ that is slightly below $\sup P_{2}$, we have $\Pi_{2}(p)=\left[1-F_{1}^{*}(p)\right] p \alpha$. If $F_{1}^{*}$ does not have a mass at $\sup P_{1}$, then $\Pi_{2}(p) \rightarrow 0$ as $p \rightarrow \sup P_{2}$, a contradiction to $\pi_{2}>0$. Further, by 
Lemma 1, the mass point in $F_{1}^{*}$ must be at $r$, and hence $\sup P_{1}=\sup P_{2}=r$. This shows that Type-I equilibrium is one possible type of equilibrium.

When $\sup P_{2}<\sup P_{1}, F_{1}^{*}$ may or may not have a mass at $r$, suggesting that both Types II and III equilibria are possible. Q.E.D.

\section{Proof of Lemma 3.}

Proof: Suppose that $(p, p+\varepsilon) \subset P_{1},(p, p+\varepsilon) \cap P_{2}=\phi$ and $(p-c, p-$ $c+\varepsilon) \cap P_{2}=\phi$. Then for $p_{1} \in(p, p+\varepsilon), \Pi_{1}\left(p_{1}\right)=\left[1-F_{2}^{*}\left(p_{1}\right)\right] p_{1}+\left[F_{2}^{*}\left(p_{1}\right)-\right.$ $\left.F_{2}^{*}\left(p_{1}-c\right)\right](1-\alpha) p_{1}$. Since $F_{2}^{*}\left(p_{1}\right)$ and $F_{2}^{*}\left(p_{1}-c\right)$ are constant on $(p, p+\varepsilon)$, $\Pi_{1}\left(p_{1}\right)$ is an increasing function over this interval, violating the equal-profit condition on $(p, p+\varepsilon) \subset P_{1}$. This proves the first statement of the Lemma. The second statement can be proved analogously. Q.E.D.

\section{Proof of Lemma 4.}

Proof: The proof proceeds by iteratively deleting dominated strategies of each seller.

We start with $S_{i}=[0, r]$ as the strategy space for each seller. By playing $p_{1}=c-\epsilon$, where $\epsilon>0$ is arbitrarily small, seller 1 can guarantee a profit $(c-\epsilon)(1-\alpha)$. Thus for seller 1 , any price $p_{1}<(c-\epsilon)(1-\alpha)$ is dominated by $p_{1}=c-\epsilon$. So we update the lower bound of seller 1's strategy space, $\underline{p_{1}}$, to $\underline{p_{1}}=(c-\epsilon)(1-\alpha)$. At price $p_{2}=\underline{p_{1}}-\epsilon$, seller 2 makes a profit of $\left(\underline{p_{1}}-\epsilon\right) \alpha$. Therefore, for seller 2 , any price $p_{2}<\left(\underline{p_{1}}-\epsilon\right) \alpha$ is dominated by price $p_{2}=p_{1}-\epsilon$, and we update the lower bound of seller 2's strategy space, $\underline{p_{2}}$, to $\underline{p_{2}}=\left(\underline{p_{1}}-\epsilon\right) \alpha$.

Now, for seller $1 p_{1}=\underline{p_{2}}+c-\epsilon$ dominates any price below $\left(\underline{p_{2}}+c-\epsilon\right)(1-\alpha)$, and for seller $2 p_{2}=\underline{p_{1}}-\epsilon$ dominates any price below $\left(\underline{p_{1}}-\epsilon\right) \alpha$. So we make the following updates: $\underline{p_{1}}=\left(\underline{p_{2}}+c-\epsilon\right)(1-\alpha)$ and $\underline{p_{2}}=\left(\underline{p_{1}}-\epsilon\right) \alpha$.

If consumers' reservation utility $r$ is sufficiently high, this iteration process will continue indefinitely, but $\left(\underline{p_{1}}, \underline{p_{2}}\right)$ will converge. Solving the two equations above simultaneously and letting $\epsilon \rightarrow 0$, we see that $\left(\underline{p_{1}}, \underline{p_{2}}\right)$ converges to $\left(\frac{c(1-\alpha)}{1-\alpha+\alpha^{2}}, \frac{c \alpha(1-\alpha)}{1-\alpha+\alpha^{2}}\right)$.

If the reservation utility $r$ is not high enough, this iteration process will 
stop as soon as $\underline{p_{2}}>r-c$. When $\underline{p_{2}}>r-c$, for seller 1 a price $p_{1}=r$ dominates $p_{1}<r(1-\alpha)$. So we have $p_{1}=r(1-\alpha)$. Then for seller 2 a price at $r(1-\alpha)$ dominates any $p_{2}<r(1-\alpha) \alpha$ and so $\underline{p_{2}}=r(1-\alpha) \alpha$. For the iteration to stop prematurely before converging to the limit $\left(\frac{c(1-\alpha)}{1-\alpha+\alpha^{2}}, \frac{c \alpha(1-\alpha)}{1-\alpha+\alpha^{2}}\right)$, we have $\underline{p_{2}}=r(1-\alpha) \alpha<\frac{c \alpha(1-\alpha)}{1-\alpha+\alpha^{2}}$, or equivalently $r<\frac{c}{1-\alpha+\alpha^{2}} \leq \frac{4 c}{3}<2 c$. Since $\sup P_{i} \leq r$, we have $\sup P_{i}-\inf P_{i}<2 c$.

Next, we continue with the case in which $\left(\underline{p_{1}}, \underline{p_{2}}\right)$ converges to $\left(\frac{c(1-\alpha)}{1-\alpha+\alpha^{2}}, \frac{c \alpha(1-\alpha)}{1-\alpha+\alpha^{2}}\right)$, i.e., $r \geq \frac{c}{1-\alpha+\alpha^{2}}$. We already know $\sup P_{2} \leq \sup P_{1}<\sup P_{2}+c$ by Lemma 2 .

First, we consider the case when $\sup P_{1}=\sup P_{2}$. When $\sup P_{1}=\sup P_{2}$, by Proposition $2, \sup P_{2}=\sup P_{1}=r$ and seller 1's strategy has a mass point at $\sup P_{1}=r$. Suppose $r>c / \alpha$. Then pricing at $p_{1}=r$ yields seller 1 a profit $r(1-\alpha)$ if $r-c<p_{2}<r$, a profit $(r-c)(1-\alpha) / 2$ if $p_{2}=r-c$, and a profit 0 otherwise, while pricing at $p_{1}=r-c$ yields seller 1 a profit $r-c$ if $r-c<p_{2}<r$, a profit $(r-c)(1-\alpha / 2)$ if $p_{2}=r-c$. Therefore, when $r>c / \alpha$, pricing at $p_{1}=r$ is a dominated strategy for seller 1 . This contradicts seller 1 's strategy having a mass at $r$. Therefore we must have $r \leq c / \alpha$. Suppose $r>c /(1-\alpha)$. When pricing at $p_{2} \in[\max (c /(1-\alpha), r-c), r)$, seller 2 earns a profit $p_{2} \alpha$ if $p_{1}>p_{2}$, a profit $p_{2} \alpha / 2$ if $p_{1}=p_{2}$, and zero otherwise. When pricing at $p_{2}-c$, seller 2 earns a profit $p_{2}-c$ if $p_{1}>p_{2}$, a profit $p_{2}(1+\alpha) / 2$ if $p_{1}=p_{2}$. Hence for seller 2 , any $p_{2} \in[\max (c /(1-\alpha), r-c), r)$ is weakly dominated by $p_{2}-c$. Therefore, we must have $r \leq c /(1-\alpha)$. Combining $r \leq c / \alpha$ and $r \leq c /(1-\alpha)$, we have $r \leq 2 c$ for any $\alpha$. Consequently, we have $\sup P_{i}-\inf P_{i}<2 c$.

Second, consider the case when $\sup P_{2}<\sup P_{1}$. If $\sup P_{2}>c / \alpha$, we can verify that, for seller 1 , any $p_{1} \in\left(\sup P_{2}, \sup P_{1}\right]$ is weakly dominated by $p_{1}-c$, which implies $\sup P_{2}=\sup P_{1}$, a contradiction. Thus, we must have $\sup P_{2} \leq c / \alpha$. Then we can verify that for seller 1 any price $p_{1} \in\left(c / \alpha, \sup P_{1}\right]$ is weakly dominated by $p_{1}-c$. This leads to $\sup P_{1} \leq c / \alpha$. If $\sup P_{2}>$ $c /(1-\alpha)$, we can verify that for seller 2 any price $p_{2} \in\left(c /(1-\alpha), \sup P_{2}\right]$ is 
weakly dominated by $p_{2}-c$. So, $\sup P_{2} \leq c /(1-\alpha)$. Since $\sup P_{1}<\sup P_{2}+c$, we also have $\sup P_{1}<c /(1-\alpha)+c$.

Because inf $P_{i} \geq \underline{p_{i}}$, we have $\sup P_{1}-\inf P_{1} \leq \min \left\{\frac{c}{\alpha}, \frac{c}{1-\alpha}+c\right\}-\frac{c(1-\alpha)}{1-\alpha+\alpha^{2}}$ and $\sup P_{2}-\inf P_{2} \leq \min \left\{\frac{c}{\alpha}, \frac{c}{1-\alpha}\right\}-\frac{c \alpha(1-\alpha)}{1-\alpha+\alpha^{2}}$. It is then easy to verify that, for any $\alpha, \sup P_{i}-\inf P_{i}<2 c$. Q.E.D.

\section{Lemma 5 below is Needed in the Proofs of Propositions 3, 4 and} 8.

Lemma $5 \Pi_{2}\left(p_{2}\right)$ is non-decreasing on $\left(0, \sup P_{2}-c\right)$.

Proof of Lemma 5: For any $p \in\left(0, \sup P_{2}-c\right)$, we can always find an $\epsilon>0$ sufficiently small so that either $(p, p+\epsilon) \subset P_{2}$ or $(p, p+\epsilon) \cap P_{2}=\varnothing$. If $(p, p+\epsilon) \subset P_{2}, \Pi_{2}$ is constant on $(p, p+\epsilon)$ by definition of a Nash equilibrium. We next show that $\Pi_{2}\left(p_{2}\right)$ increases on $(p, p+\epsilon)$ if $(p, p+\epsilon) \cap P_{2}=\varnothing$.

Suppose $(p, p+\epsilon) \cap P_{2}=\varnothing$. By Lemma 4, sup $P_{2}-\inf P_{2}<2 c$. We then have $(p-c, p-c+\epsilon) \cap P_{2}=\varnothing$, since the current step deals with the case in which $p<\sup P_{2}-c$. Therefore, $\Pi_{1}\left(p_{1}\right)$ increases on $(p, p+\epsilon)$, which implies that $(p, p+\epsilon) \cap P_{1}=\varnothing$. Consider the following two cases.

If $(p+c, p+c+\epsilon) \cap P_{1}=\varnothing$, then $\Pi_{2}\left(p_{2}\right)$ increases on $(p, p+\epsilon)$.

If $(p+c, p+c+\epsilon) \subset P_{1}$, then we have $(p+c, p+c+\epsilon) \subset P_{2}$ by Lemma 3 , since $(p, p+\epsilon) \cap P_{2}=\varnothing$ by assumption. Invoking the equal-profit condition for seller 2 on the interval $(p+c, p+c+\epsilon) \subset P_{2}$, we have $\pi_{2}=\alpha p\left(1-F_{1}^{*}(p)\right)$ or $F_{1}^{*}(p)=1-\pi_{2} /(\alpha p)$. For any $p^{\prime} \in(p, p+\epsilon)$, we then have

$$
\begin{aligned}
\Pi_{2}\left(p^{\prime}\right) & =p^{\prime}\left[1-F_{1}^{*}\left(p^{\prime}+c\right)\right]+\alpha p^{\prime}\left[F_{1}^{*}\left(p^{\prime}+c\right)-F_{1}^{*}\left(p^{\prime}\right)\right] \\
& =p^{\prime}\left[1-\alpha F_{1}^{*}\left(p^{\prime}\right)\right]-p^{\prime}(1-\alpha) F_{1}^{*}\left(p^{\prime}+c\right) \\
& =p^{\prime}\left[1-\alpha F_{1}^{*}\left(p^{\prime}\right)\right]-p^{\prime}(1-\alpha)\left(1-\frac{\pi_{2}}{\alpha\left(p^{\prime}+c\right)}\right) \\
& \left.=\alpha p^{\prime}\left[1-F_{1}^{*}\left(p^{\prime}\right)\right]+\frac{\pi_{2}(1-\alpha) p^{\prime}}{\alpha\left(p^{\prime}+c\right)}\right),
\end{aligned}
$$

which is an increasing function on $(p, p+\varepsilon)$. Therefore, in both cases, $\Pi_{2}\left(p_{2}\right)$ increases on $(p, p+\epsilon)$ if $(p, p+\epsilon) \cap P_{2}=\varnothing$. Q.E.D. 


\section{Proof of Proposition 3.}

Proof: We identify the only equilibrium satisfying $\sup P_{1}=\sup P_{2}$. As shown in the proof of Proposition 2 above, when $\sup P_{1}=\sup P_{2}$, we have $\sup P_{1}=\sup P_{2}=r$ and $F_{1}^{*}$ has a mass at $r$. The remainder of the proof proceeds in 3 steps.

Step 1. We show inf $P_{2} \geq \sup P_{2}-c$.

First, we prove that $\exists \epsilon>0$, such that $\left(\sup P_{2}-c-\epsilon, \sup P_{2}-c\right) \cap P_{1}=\varnothing$.

By the proof of Lemma 3, we know that if $F_{1}^{*}$ has a mass at $r$, then $\exists \delta>0$ such that $[r-c, r-c+\delta) \cap P_{2}=\varnothing$. Since sup $P_{i}-\inf P_{i}<2 c$, we can choose $\delta$ small enough so that $[r-2 c, r-2 c+\delta) \cap P_{2}=\varnothing$. Thus, $\Pi_{1}(p)$ increases on $[r-c, r-c+\delta)$.

Since $\Pi_{1}(p)$ is a continuous increasing function on $[r-c, r-c+\delta)$, we have $\Pi_{1}(p)<\pi_{1}$ on $[r-c, r-c+\delta)$. By the general continuity of $\Pi_{1}(p)$, we can find a $\epsilon$ small enough such that $\forall p \in(r-c-\epsilon, r-c), \Pi_{1}(p)<\pi_{1}$, which implies that $(r-c-\epsilon, r-c) \cap P_{1}=\varnothing$.

Second, we prove that $\exists \epsilon^{\prime}>0$, such that $\left(\sup P_{2}-c-\epsilon^{\prime}\right.$, sup $\left.P_{2}-c\right) \cap P_{2}=$ $\varnothing$.

We know there exists $\epsilon^{\prime}>0$ such that $\sup P_{2}-\epsilon^{\prime}>\sup P_{1}-c$ and $\left(\sup P_{2}-\epsilon^{\prime}, \sup P_{2}\right) \subset P_{2}$ for the very existence of $\sup P_{2}$. We then have $\left(\sup P_{2}-\epsilon^{\prime}, \sup P_{2}\right) \subset P_{1}$ by Lemma 3 . It follows from the equal-profit condition for firm 2 that $F_{1}^{*}(p)=1-\pi_{2} /(\alpha p)$ on $\left(\sup P_{2}-\epsilon^{\prime}, \sup P_{2}\right)$.

We already have $\left(\sup P_{2}-c-\epsilon, \sup P_{2}-c\right) \cap P_{1}=\varnothing$. Let $\epsilon^{\prime}<\epsilon$, so $\left(\sup P_{2}-c-\epsilon^{\prime}, \sup P_{2}-c\right) \cap P_{1}=\varnothing$.

On $\left(\sup P_{2}-c-\epsilon^{\prime}, \sup P_{2}-c\right)$, by the equal-profit condition, we have $\Pi_{2}(p)=p\left(1-F_{1}^{*}(p+c)\right)+\alpha p\left(F_{1}^{*}(p+c)-F_{1}^{*}(p)\right)=\alpha p\left(1-F_{1}^{*}(p)\right)+\frac{\pi_{2}(1-\alpha) p}{\alpha(p+c)}$, which is an increasing function on $\left(\sup P_{2}-c-\epsilon^{\prime}, \sup P_{2}-c\right)$, where $F_{1}^{*}(p)$ is constant. This implies $\left(\sup P_{2}-c-\epsilon^{\prime}, \sup P_{2}-c\right) \cap P_{2}=\varnothing$.

Third, by Lemma $5 \Pi_{2}(p)$ is non-decreasing when $p<\sup P_{2}-c$. The above result implies that $\forall p<\sup P_{2}-c, \Pi_{2}(p)<\pi_{2}$, and thus $p \notin P_{2}$. Hence inf $P_{2} \geq \sup P_{2}-c=r-c$. 
Step 2. We next show that $P_{1}$ and $P_{2}$ are convex. For any $(p, p+\varepsilon) \subset P_{1}$, by Lemma 3 we must have $(p, p+\varepsilon) \subset P_{2}$ since we have just shown that inf $P_{2} \geq r-c$ in Step 1. Likewise, for any $(p, p+\varepsilon) \subset P_{2}$, we must also have $(p, p+\varepsilon) \subset P_{1}$. Therefore, $C l\left(P_{1}\right)=C l\left(P_{2}\right)$, where $C l(P)$ is the closure of set $P$, and hence $\inf P_{1}=\inf P_{2}$. Now suppose $(p, p+\varepsilon) \cap P_{i}=\varnothing$ for some $p>r-c$. Then we must have $(p, p+\varepsilon) \cap P_{j}=\varnothing$, which implies that $\Pi_{i}(p)$ is increasing on $(p, p+\varepsilon)$. However, Lemma 1 implies that $\Pi_{1}(p)$ is always continuous and $\Pi_{2}(p)$ is also continuous on $(r-c, r)$. We thus have reached a contradiction to the equal-profit condition of an equilibrium. Therefore, there can not be any hole in $P_{i}$, i.e., $P_{i}$ is convex, $i=1,2$.

Step 3. We derive the exact form of the equilibrium using the equal-profit condition. For $p \in P_{1}$, we have $\Pi_{1}(p)=p\left[1-F_{2}^{*}(p)\right]+(1-\alpha) p F_{2}^{*}(p)=p[1-$ $\left.\alpha F_{2}^{*}(p)\right]$. At $p=r$, we obtain $\pi_{1}=r(1-\alpha)$. So $F_{2}^{*}(p)=(1 / \alpha)[1-r(1-\alpha) / p]$. Setting $F_{2}^{*}(p)=0$ gives $\inf P_{1}=\inf P_{2}=r(1-\alpha)$. We also have $\Pi_{2}(p)=$ $\alpha p\left[1-F_{1}^{*}(p)\right]$, for $p \in P_{2}$. Let $p \rightarrow r(1-\alpha)$, we have $\pi_{2}=r(1-\alpha) \alpha$, and hence $F_{1}^{*}(p)=1-r(1-\alpha) / p$.

Lastly, for the above to be an equilibrium, any price below $r-c$ must be a dominated strategy for seller 2 , i.e., $\Pi_{2}(r-c-\varepsilon)<r(1-\alpha) \alpha$. When $\varepsilon \rightarrow 0$, this inequality becomes $(r-c)(1-\alpha)+(r-c) \alpha^{2}<r(1-\alpha) \alpha$, or equivalently, $r / c<s(\alpha) \equiv\left(1-\alpha+\alpha^{2}\right) /\left(1-2 \alpha+2 \alpha^{2}\right)$. This gives the condition on the parameters for Type-I Equilibrium to hold. Furthermore, from the above construction process, it is evident that Type-I Equilibrium is the only equilibrium under this condition. Q.E.D.

\section{Comparative Statics in Equilibrium II.}

The expected prices charged by the sellers are $E p_{1}=\frac{\pi_{2}}{\alpha} \ln \left(\frac{\alpha \pi_{1}}{(1-\alpha) \pi_{2}}\right)+$ $\frac{\pi_{2}}{1-\alpha} \ln \left(\frac{(1-\alpha)(r-c)}{\pi_{1}-c(1-\alpha)}\right)+\frac{c \pi_{2}}{\pi_{1}-c(1-\alpha)}+\frac{\pi_{2}-r \alpha}{1-\alpha}$ and $E p_{2}=\frac{\pi_{1}}{\alpha} \ln \left(\frac{\alpha \pi_{1}}{(1-\alpha) \pi_{2}}\right)+\frac{\pi_{1}}{1-\alpha} \ln \left(\frac{r(1-\alpha)}{\pi_{1}}\right)+$ $\frac{c \pi_{1}}{r(1-\alpha)}-c$. The expected price a loyal consumer pays is

$$
E p^{l}=\int_{\frac{\pi_{1}}{1-\alpha}}^{r} x\left(1-F_{2}^{*}(x-c)\right) d F_{1}^{*}(x)+\int_{\frac{\pi_{1}}{1-\alpha}-c}^{r-c} x\left(1-F_{1}^{*}(x+c)\right) d F_{2}^{*}(x)+
$$

$\int_{\frac{\pi_{2}}{\alpha}}^{\frac{\pi_{1}}{1-\alpha}} x d F_{1}^{*}(x)+r\left(1-F_{2}^{*}(r-c)\right)\left(\frac{\pi_{2}-\alpha(r-c)}{(1-\alpha)(r-c)}\right)$. The expected price a switcher pays is 


$$
\begin{aligned}
& E p^{s}=\int_{\frac{\pi_{1}}{1-\alpha}-c}^{r-c} x d F_{2}^{*}(x)+\int_{\frac{\pi_{2}}{\alpha}}^{\frac{\pi_{1}}{1-\alpha}} x d G(x)=\frac{\pi_{1}}{1-a} \ln \left(\frac{r(1-\alpha)}{\pi_{1}}\right)-c+\frac{c \pi_{1}}{r(1-\alpha)}- \\
& \frac{\pi_{2}(1-\alpha)}{\alpha^{2}} \ln \left(\frac{\alpha \pi_{1}}{(1-\alpha) \pi_{2}}\right)+\frac{2}{\alpha^{2}}\left(\alpha \pi_{1}-(1-\alpha) \pi_{2}\right) \text {, where } G(x)=1-\left(1-F_{1}^{*}(x)\right)(1- \\
& \left.F_{2}^{*}(x)\right) .
\end{aligned}
$$

\section{Comparative Statics in Equilibrium III.}

The expected prices charged by the two sellers are $E p_{1}=\frac{\pi_{2}}{\alpha} \ln \left(\frac{\alpha \pi_{1}}{(1-\alpha) \pi_{2}}\right)+$ $\frac{\pi_{2}}{1-\alpha} \ln \left(\frac{(1-\alpha) \pi_{2}}{\alpha\left(\pi_{1}-c(1-\alpha)\right)}\right)+\frac{c \pi_{2}}{\pi_{1}-c(1-\alpha)}-\frac{c \alpha}{1-\alpha}$ and $E p_{2}=\frac{\pi_{1}}{\alpha} \ln \left(\frac{\alpha \pi_{1}}{(1-\alpha) \pi_{2}}\right)+\frac{\pi_{1}}{1-\alpha} \ln \left(\frac{(1-\alpha)\left(\pi_{2}+c \alpha\right)}{\alpha \pi_{1}}\right)+$ $\frac{c \alpha \pi_{1}}{(1-\alpha)\left(\pi_{2}+c \alpha\right)}-c$. On average a loyal consumer pays

$$
E p^{l}=\int_{\frac{\pi_{1}}{1-\alpha}}^{\frac{\pi_{2}}{\alpha}+c} x\left(1-F_{2}^{*}(x-c)\right) d F_{1}^{*}(x)+\int_{\frac{\pi_{1}}{1-\alpha}-c}^{\frac{\pi_{2}}{\alpha}} x\left(1-F_{1}^{*}(x+c)\right) d F_{2}^{*}(x)+
$$

$\int_{\frac{\pi_{2}}{\alpha}}^{\frac{\pi_{1}}{1-\alpha}} x d F_{1}^{*}(x)$. On average a switcher pays

$$
E p^{s}=\int_{\frac{\pi_{1}}{1-\alpha}-c}^{\frac{\pi_{2}}{\alpha}} x d F_{2}^{*}(x)+\int_{\frac{\pi_{2}}{\alpha}}^{\frac{\pi_{1}}{1-\alpha}} x d G(x)=\frac{\pi_{1}}{1-a} \ln \left(\frac{\left(\pi_{2}+c \alpha\right)(1-\alpha)}{\alpha \pi_{1}}\right)-c+\frac{c \alpha \pi_{1}}{\left(\pi_{2}+c \alpha\right)(1-\alpha)}-
$$
$\frac{\pi_{2}(1-\alpha)}{\alpha^{2}} \ln \left(\frac{\alpha \pi_{1}}{(1-\alpha) \pi_{2}}\right)+\frac{2}{\alpha^{2}}\left(\alpha \pi_{1}-(1-\alpha) \pi_{2}\right)$, where $G(x)=1-\left(1-F_{1}^{*}(x)\right)(1-$ $\left.F_{2}^{*}(x)\right)$.

\section{Lemmas Needed in the Proofs of Propositions 4 and 8.}

Lemma $6 \inf P_{2} \geq \sup P_{2}-c$.

Proof of Lemma 6: First, we prove that $\exists \epsilon>0$, such that $\left(\sup P_{2}-c-\right.$ $\left.\epsilon, \sup P_{2}-c\right) \cap P_{1}=\varnothing$.

We know that there must exist $\delta>0$ such that $\left(\sup P_{2}, \sup P_{2}+\delta\right) \subset P_{1}$ (otherwise seller 2 could increase its profit by charging a price slightly above $\sup P_{2}$, a contradiction). Applying the equal-profit condition for seller 1 on $\left(\sup P_{2}, \sup P_{2}+\delta\right)$, we have $\pi_{1}=\left[1-F_{2}^{*}(p-c)\right](1-\alpha) p$ or $F_{2}^{*}(p-c)=$ $1-\frac{\pi_{1}}{(1-\alpha) p}$. Since $\sup P_{2}-\inf P_{2}<2 c$ (from Lemma 4), we let $\delta$ be sufficiently small so that $\left(\sup P_{2}-2 c, \sup P_{2}-2 c+\delta\right) \cap P_{2}=\varnothing$. On $\left(\sup P_{2}-c, \sup P_{2}-\right.$ $c+\delta), \Pi_{1}\left(p_{1}\right)=p_{1}\left[1-\alpha F_{2}^{*}\left(p_{1}\right)\right]=p_{1}-\alpha p_{1}\left(1-\frac{\pi_{1}}{(1-\alpha)\left(p_{1}+c\right)}\right)=p_{1}(1-$ $\alpha)+\frac{\alpha \pi_{1}}{1-\alpha}\left(1-\frac{c}{p_{1}+c}\right)$, which is an increasing function on this open interval. Therefore, $\left(\sup P_{2}-c, \sup P_{2}-c+\delta\right) \cap P_{1}=\varnothing$.

Recall that $\Pi_{1}\left(p_{1}\right)$ is a continuous function (see discussions after Lemma $1)$. We then have $\Pi_{1}\left(\sup P_{2}-c\right)<\pi_{1}$, and can find an $\epsilon>0$ small enough so that $\Pi_{1}\left(p_{1}\right)<\pi_{1}$ on $\left(\sup P_{2}-c-\epsilon, \sup P_{2}-c\right)$, i.e., $\left(\sup P_{2}-c-\epsilon, \sup P_{2}-\right.$ c) $\cap P_{1}=\varnothing$. 
Second, we prove that $\exists \epsilon^{\prime}>0$, such that $\left(\sup P_{2}-c-\epsilon^{\prime}\right.$, sup $\left.P_{2}-c\right) \cap P_{2}=$ $\varnothing$.

By Lemma 2, we have $\sup P_{1}-c<\sup P_{2}$. Therefore, there must exist an $\epsilon^{\prime}>0$ small enough so that $\sup P_{2}-\epsilon^{\prime}>\sup P_{1}-c$ and that $\left(\sup P_{2}-\right.$ $\left.\epsilon^{\prime}, \sup P_{2}\right) \subset P_{2}$ since there is no mass in $F_{2}^{*}$ by Lemma 1 .

We then must have $\left(\sup P_{2}-\epsilon^{\prime}, \sup P_{2}\right) \subset P_{1}$, or $\Pi_{2}\left(p_{2}\right)$ would be increasing on $\left(\sup P_{2}-\epsilon^{\prime}, \sup P_{2}\right)$. Applying the equal profit condition for firm 2 on $\left(\sup P_{2}-\epsilon^{\prime}, \sup P_{2}\right)$, we have $\pi_{2}=\alpha p_{2}\left[1-F_{1}^{*}\left(p_{2}\right)\right]$ or $F_{1}^{*}\left(p_{2}\right)=1-\pi_{2} /\left(\alpha p_{2}\right)$.

We have shown that $\exists \epsilon>0$ so that $\left(\sup P_{2}-c-\epsilon, \sup P_{2}-c\right) \cap P_{1}=\varnothing$. Let $\epsilon^{\prime}<\epsilon$. Then $\left(\sup P_{2}-c-\epsilon^{\prime}, \sup P_{2}-c\right) \cap P_{1}=\varnothing$, and hence $F_{1}^{*}$ remains constant on $\left(\sup P_{2}-c-\epsilon^{\prime}, \sup P_{2}-c\right)$.

On $\left(\sup P_{2}-c-\epsilon^{\prime}, \sup P_{2}-c\right)$, we have $\Pi_{2}\left(p_{2}\right)=p_{2}\left(1-F_{1}^{*}\left(p_{2}+c\right)\right)+$ $\alpha p_{2}\left[F_{1}^{*}\left(p_{2}+c\right)-F_{1}^{*}\left(p_{2}\right)\right]=\alpha p_{2}\left[1-F_{1}^{*}\left(p_{2}\right)\right]+\frac{\pi_{2}(1-\alpha) p_{2}}{\alpha\left(p_{2}+c\right)}$, which is an increasing function on this open interval. This implies $\left(\sup P_{2}-c-\epsilon^{\prime}, \sup P_{2}-c\right) \cap P_{2}=$ $\varnothing$.

Since we have shown in Lemma 5 that $\Pi_{2}\left(p_{2}\right)$ is non-decreasing when $p_{2}<\sup P_{2}-c, \Pi_{2}\left(p_{2}\right)$ being increasing on $\left(\sup P_{2}-c-\epsilon^{\prime}, \sup P_{2}-c\right)$ implies that for $p_{2}<\sup P_{2}-c, \Pi_{2}\left(p_{2}\right)<\pi_{2}$ and therefore $p_{2} \notin P_{2}$. This establishes $\inf P_{2} \geq \sup P_{2}-c$. Q.E.D.

Lemma $7 \inf P_{1} \geq \sup P_{1}-c$.

Proof of Lemma 7: First, we want to show that $\Pi_{1}$ is nondecreasing on (inf $\left.P_{2}, \sup P_{1}-c\right)$. Pick any $\epsilon>0$ and $p$ so that $(p, p+\epsilon) \subset\left(\inf P_{2}, \sup P_{1}-c\right)$. If $(p, p+\epsilon) \subset P_{1}, \Pi_{1}\left(p_{1}\right)=\pi_{1}$ is constant on $(p, p+\epsilon)$ by definition of an equilibrium. We next show that if $(p, p+\epsilon) \cap P_{1}=\varnothing, \Pi_{1}\left(p_{1}\right)$ increases on $(p, p+\epsilon)$.

Suppose $(p, p+\epsilon) \cap P_{1}=\varnothing$. If $(p, p+\epsilon) \cap P_{2}=\varnothing$, then clearly $\Pi_{1}\left(p_{1}\right)$ is an increasing function on $(p, p+\epsilon)$. If $(p, p+\epsilon) \subset P_{2}$, by Lemma 3 , $(p+c, p+c+\epsilon) \subset P_{1}$ since $(p, p+\epsilon) \cap P_{1}=\varnothing$ by assumption. Then, by the equal-profit condition for firm 1 , for $p_{1} \in(p+c, p+c+\epsilon)$ we have $\pi_{1}=\left[1-F_{2}^{*}\left(p_{1}-c\right)\right](1-\alpha) p_{1}$ or $F_{2}^{*}\left(p_{1}-c\right)=1-\pi_{1} /\left((1-\alpha) p_{1}\right)$. This implies 
$F_{2}^{*}\left(p_{1}\right)=1-\pi_{1} /\left[(1-\alpha)\left(p_{1}+c\right)\right], \forall p_{1} \in(p, p+\epsilon)$. Therefore, $\forall p_{1} \in(p, p+\epsilon)$, $\Pi_{1}\left(p_{1}\right)=p_{1}\left[1-F_{2}^{*}\left(p_{1}\right)\right]+p_{1}(1-\alpha)\left[F_{2}^{*}\left(p_{1}\right)-F_{2}^{*}\left(p_{1}-c\right)\right]=p_{1}-\alpha p_{1} F_{2}^{*}\left(p_{1}\right)=$ $(1-\alpha) p_{1}+\frac{\pi_{1} \alpha p_{1}}{(1-\alpha)\left(p_{1}+c\right)}$, which is an increasing function on this interval.

Second, we show $\forall \epsilon>0$, (sup $\left.P_{1}-c-\epsilon, \sup P_{1}-c\right) \cap P_{1}=\varnothing$. Suppose otherwise. Then $\exists \epsilon>0$ so that $\left(\sup P_{1}-c-\epsilon, \sup P_{1}-c\right) \subset P_{1}$. Note that $\left(\sup P_{1}-2 c-\epsilon, \sup P_{1}-2 c\right) \cap P_{2}=\varnothing$ since $\sup P_{1}-c<\sup P_{2}$ (by Lemma 2) and inf $P_{2} \geq \sup P_{2}-c$ as shown in Lemma 6. Therefore, $\left(\sup P_{1}-c-\epsilon, \sup P_{1}-c\right) \subset P_{2}$ by Lemma 3 . Invoking the equal-profit condition for firm 1 , on $\left(\sup P_{1}-c-\epsilon, \sup P_{1}-c\right)$, we have $\pi_{1}=p_{1}-\alpha p_{1} F_{2}^{*}\left(p_{1}\right)$ or $F_{2}^{*}\left(p_{1}\right)=(1 / \alpha)\left[1-\pi_{1} / p_{1}\right]$. Then $\forall p_{1} \in\left(\sup P_{1}-\epsilon, \sup P_{1}\right), \Pi_{1}\left(p_{1}\right)=(1-$ a) $p_{1}\left(1-F_{2}^{*}\left(p_{1}-c\right)\right)=-\frac{p_{1}(1-\alpha)^{2}}{\alpha}+\frac{\pi_{1}(1-\alpha)}{\alpha}\left(1+\frac{c}{p_{1}-c}\right)$, which is a decreasing function over this interval. This implies that $\left(\sup P_{1}-\epsilon, \sup P_{1}\right) \cap P_{1}=\varnothing$. Therefore, the only remaining possibility is $\sup P_{1} \in P_{1}$. In such a case, however, $\Pi_{1}\left(p_{1}\right)$ being decreasing on $\left(\sup P_{1}-\epsilon, \sup P_{1}\right)$ would contradict the continuity of $\Pi_{1}$. Therefore, $\exists \epsilon>0$ so that $\left(\sup P_{1}-c-\epsilon\right.$, sup $\left.P_{1}-c\right) \cap P_{1}=\varnothing$. We have shown above that $\Pi_{1}\left(p_{1}\right)$ is nondecreasing on (inf $\left.P_{2}, \sup P_{1}-c\right)$. Therefore, $p_{1} \notin P_{1}$ if $p_{1}<\sup P_{1}-c$. This show that inf $P_{1} \geq \sup P_{1}-c$. Q.E.D.

Lemma $8 P_{1}, P_{1} \cap P_{2}$ and $P_{2}-P_{1} \cap P_{2}$ are convex sets.

Proof of Lemma 8: Consider any interval $(p, p+\epsilon) \subset\left(\inf P_{1}, \sup P_{2}\right)$. Since we have seen $\sup P_{i}-\inf P_{i} \leq c, i=1,2$, in Lemmas 6 and 7 , if $(p, p+\epsilon) \subset P_{i}$, then $(p, p+\epsilon) \subset P_{j}$ by Lemma 3. So, if $(p, p+\epsilon) \cap P_{i}=\varnothing$, then $(p, p+\epsilon) \cap P_{j}=\varnothing$, and $\Pi_{j}\left(p_{j}\right)$ is increasing on $(p, p+\epsilon)$. Therefore, if $(p, p+\epsilon) \cap P_{i}=\varnothing$, then $p$ must be smaller than inf $P_{1}$. This shows that there can not be any hole in $\left(\inf P_{1}, \sup P_{2}\right)$, and thus $P_{1} \cap P_{2}=\left(\inf P_{1}, \sup P_{2}\right)$ is convex.

By Lemma 3, $\forall(p, p+\epsilon) \subset\left(\sup P_{2}, \sup P_{1}\right)$, if $(p, p+\epsilon) \subset P_{1}$, then $(p-c, p-c+\epsilon) \subset P_{2} . \forall(p, p+\epsilon) \subset\left(\sup P_{2}-c, \sup P_{1}-c\right)$, and if $(p, p+\epsilon) \subset P_{2}$, then $(p+c, p+c+\epsilon) \subset P_{1}$. If there is a hole in $\left(\sup P_{2}, \sup P_{1}\right)$, i.e. $\exists(p, p+\epsilon) \subset$ $\left(\sup P_{2}, \sup P_{1}\right)$ such that $(p, p+\epsilon) \cap P_{1}=\varnothing$, then $(p-c, p-c+\epsilon) \cap P_{2}=$ 
$\varnothing$. Thus, $\Pi_{1}\left(p_{1}\right)$ is increasing on such a hole $(p, p+\epsilon)$. Since $\Pi_{1}\left(p_{1}\right)$ is continuous, we can conclude that no hole exists in $\left(\sup P_{2}, \sup P_{1}\right)$. Thus $\left(\sup P_{2}, \sup P_{1}\right) \subset P_{1}$ is convex. Combining this with $\left(\inf P_{1}, \sup P_{2}\right)$ being convex, we see that $P_{1}$ is convex.

Since $\left(\sup P_{2}, \sup P_{1}\right) \subset P_{1}$ is convex, by Lemma $3 P_{2}-P_{1} \cap P_{2}=\left(\sup P_{2}-\right.$ $\left.c, \sup P_{1}-c\right) \subset P_{2}$, which is also convex. From this, we also have inf $P_{2}=$ $\sup P_{2}-c$. Q.E.D.

Note: Lemmas 6-8 do not depend on whether seller 1's strategy has a mass at $\sup P_{1}=r$ or not. So they are equally valid for deriving Types II and III Equilibria.

\section{Proof of Proposition 4.}

Proof: We are now ready to derive the exact form of Type-II equilibrium, i.e., the type of equilibrium in which seller 1's strategy has a mass at $r$.

$F_{1}^{*}$ having a mass at $\sup P_{1}=r$ implies that $\exists \epsilon>0$ so that $(r-c, r-$ $c+\epsilon) \cap P_{2}=\varnothing$. We thus have the following characterization about $P_{2}$ : $C l\left(P_{2}\right)=C l\left(\left(\sup P_{2}-c, r-c\right)\right) \cup C l\left(\left(\inf P_{1}\right.\right.$, sup $\left.\left.P_{2}\right)\right)$, where $C l(P)$ is the closure of set $P$.

By charging sup $P_{2}$, seller 1 receives an expected payoff of $\pi_{1}=(1-$ $\alpha) \sup P_{2}$. So $\sup P_{2}=\pi_{1} /(1-\alpha)$. By charging a price arbitrarily close to inf $P_{1}$ from above, seller 2 obtains an expected payoff of $\pi_{2}=\alpha \inf P_{1}$, and thus inf $P_{1}=\pi_{2} / \alpha$.

By the equal-profit condition for seller 1 , for $p \in\left[\pi_{2} / \alpha, \pi_{1} /(1-\alpha)\right)$, we have $\pi_{1}=p-\alpha p F_{2}^{*}(p)$ or $F_{2}^{*}(p)=\frac{1}{\alpha}\left(1-\frac{\pi_{1}}{p}\right)$. For $p \in\left[\pi_{1} /(1-\alpha), r\right)$, we have $\pi_{1}=(1-\alpha) p\left[1-F_{2}^{*}(p-c)\right]$, which implies that for $p \in\left[\pi_{1} /(1-\alpha)-\right.$ $c, r-c), F_{2}^{*}(p)=1-\frac{\pi_{1}}{(1-\alpha)(p+c)}$.

By the equal-profit condition for seller 2 , for $p \in\left[\pi_{1} /(1-\alpha)-c, r-c\right)$, we have $\pi_{2}=p\left[1-F_{1}^{*}(p+c)\right]+\alpha p F_{1}^{*}(p+c)$, which implies that for $p \in$ $\left[\pi_{1} /(1-\alpha), r\right), F_{1}^{*}(p)=\frac{1}{1-\alpha}\left(1-\frac{\pi_{2}}{p-c}\right)$. For $p \in\left[\pi_{2} / \alpha, \pi_{1} /(1-\alpha)\right)$, we have $\pi_{2}=\alpha p\left[1-F_{1}^{*}(p)\right]$ or $F_{1}^{*}(p)=1-\frac{\pi_{2}}{\alpha p}$.

Combining the above equations gives the distribution functions in the 
statement of the proposition.

Lastly, to establish the existence of a unique Type-II Equilibrium, we only have to show that there exists a unique pair of $\pi_{1}, \pi_{2}>0$ in the above probability distributions.

Since both $F_{1}^{*}$ and $F_{2}^{*}$ are continuous at any point below $r$ (In Type-II Equilibrium the only mass point in $F_{1}^{*}$ occurs at $r$ ), we have

$$
\begin{aligned}
1-\frac{\pi_{2}}{\alpha \frac{\pi_{1}}{1-\alpha}} & =\frac{1}{1-\alpha}\left(1-\frac{\pi_{2}}{\frac{\pi_{1}}{1-\alpha}-c}\right), \text { and } \\
1-\frac{\pi_{1}}{r(1-\alpha)} & =\frac{1}{\alpha}\left(1-\frac{\pi_{1}}{\frac{\pi_{2}}{\alpha}}\right) .
\end{aligned}
$$

The solution to this system of equations is unique:

$$
\begin{aligned}
& \pi_{1}=\frac{c(1-\alpha)}{2 \alpha^{2}}\left(\alpha^{2}-\theta(1-\alpha)^{2}+\sqrt{\left(\alpha^{2}-\theta(1-\alpha)^{2}\right)^{2}+4 \alpha^{2} \theta(1-\alpha)}\right) \\
& \pi_{2}=\frac{1}{\frac{1-\alpha}{\alpha \pi_{1}}+\frac{1}{r(1-\alpha)}}
\end{aligned}
$$

where $\theta \equiv r / c$. Thus, the Type-II Equilibrium we have just identified is indeed well defined and unique.

For Type-II Equilibrium to hold, we must have these restrictions on the strategy sets: $\pi_{1} /(1-\alpha)<r$ and $r-c<\pi_{2} / \alpha$, from which the parameter conditions in the Proposition follow: $\frac{1-\alpha+\alpha^{2}}{(1-\alpha)^{2}+\alpha^{2}} \equiv s(\alpha)<\frac{r}{c}<t(\alpha) \equiv$ $\frac{1}{3\left(1-3 \alpha+3 \alpha^{2}\right)}\left(7 \alpha^{2}-7 \alpha+2-\frac{2^{\frac{1}{3}} h}{k}+\frac{k}{2^{\frac{1}{3}}}\right)$, where $h=-4+19 \alpha-35 \alpha^{2}+26 \alpha^{3}-4 \alpha^{4}$, $k=\left(\begin{array}{c}16-114 \alpha+342 \alpha^{2}-521 \alpha^{3}+387 \alpha^{4}-87 \alpha^{5}-16 \alpha^{6}+ \\ \sqrt{3} \alpha\left(3\left(1-3 \alpha+3 \alpha^{2}\right)\right) \sqrt{-4+36 \alpha-112 \alpha^{2}+168 \alpha^{3}-117 \alpha^{4}+32 \alpha^{5}}\end{array}\right)^{\frac{1}{3}}$. Q.E.D.

\section{Proof of Proposition 8.}

Proof: To a large extent, the derivation of Type-III Equilibrium proceeds in parallel to that of Type-II Equilibrium. The only difference is that we now examine the case in which seller 1's strategy has no mass point. 
When seller 1's strategy has no mass point, there can be no hole in $P_{2}$, i.e., $P_{2}$ is convex. From Lemma 8 , we know $P_{1}$ is convex. In addition, $\inf P_{1}=\sup P_{1}-c$.

By charging $\sup P_{2}$, seller 1 receives an expected payoff of $\pi_{1}=(1-$ $\alpha) \sup P_{2}$. So $\sup P_{2}=\pi_{1} /(1-\alpha)$. By charging a price arbitrarily close to $\inf P_{1}$ from above, seller 2 obtains an expected payoff of $\pi_{2}=\alpha \inf P_{1}$, and thus inf $P_{1}=\pi_{2} / \alpha$.

Applying the equal-profit condition for seller 2 on $\left[\pi_{1} /(1-\alpha)-c, \pi_{2} / \alpha\right)$, that for seller 1 on $\left[\pi_{1} /(1-\alpha), \pi_{2} / \alpha+c\right)$, and those for both sellers on $\left[\pi_{2} / \alpha, \pi_{1} /(1-\alpha)\right)$ readily leads to the exact form of the distribution functions as given in the statement of this proposition.

We still need to find a unique pair $\pi_{1}, \pi_{2}>0$ satisfying the probability distributions. Since neither firm's strategy has a probability mass, both $F_{1}^{*}$ and $F_{2}^{*}$ are continuous in Type-III Equilibrium. We thus have

$$
\begin{aligned}
1-\frac{\pi_{2}}{\alpha \frac{\pi_{1}}{1-\alpha}} & =\frac{1}{1-\alpha}\left(1-\frac{\pi_{2}}{\frac{\pi_{1}}{1-\alpha}-c}\right), \\
1-\frac{\pi_{1}}{\left(\frac{\pi_{2}}{\alpha}+c\right)(1-\alpha)} & =\frac{1}{\alpha}\left(1-\frac{\pi_{1}}{\frac{\pi_{2}}{\alpha}}\right) .
\end{aligned}
$$

This system of equations has a unique positive solution: $\pi_{1}=\frac{c(1-\alpha)^{2} t(\alpha)[t(\alpha)-1]}{\alpha+(1-2 \alpha) t(\alpha)}$ and $\pi_{2}=c \alpha(t(\alpha)-1)$, where $\theta \equiv r / c$ and $t(\alpha)$ is as defined in Proposition 4. Lastly, for Type-III equilibrium to hold requires $\sup P_{1}=\pi_{2} / \alpha+c \leq r$, or $r / c \geq t(\alpha)$. Q.E.D.

\section{Proof of Proposition 9.}

Proof: Since neither firm's strategy has a mass point in Type-III equilibrium, each firm's average promotional depth is simply

$$
\sup P_{i}-E\left[p_{i} \mid p_{i}<\sup P_{i}\right]=\sup P_{i}-E p_{i} \text {, for } i=1,2 .
$$

From Proposition 8, we see that both $\pi_{1}$ and $\pi_{2}$ are multiplicatively sep- 
arable and linear in $c$. Therefore, both $\sup P_{1}=\pi_{2} / \alpha+c$ and $\sup P_{2}=$ $\pi_{1} /(1-\alpha)$ are multiplicatively separable and linear in $c$. We can also readily check that both $E p_{1}$ and $E p_{2}$ are also multiplicatively separable and lin-

ear in $c$, where $E p_{1}=\frac{\pi_{2}}{\alpha} \ln \left(\frac{\alpha \pi_{1}}{(1-\alpha) \pi_{2}}\right)+\frac{\pi_{2}}{1-\alpha} \ln \left(\frac{(1-\alpha) \pi_{2}}{\alpha\left(\pi_{1}-c(1-\alpha)\right)}\right)+\frac{c \pi_{2}}{\pi_{1}-c(1-\alpha)}-\frac{c \alpha}{1-\alpha}$ and $E p_{2}=\frac{\pi_{1}}{\alpha} \ln \left(\frac{\alpha \pi_{1}}{(1-\alpha) \pi_{2}}\right)+\frac{\pi_{1}}{1-\alpha} \ln \left(\frac{(1-\alpha)\left(\pi_{2}+c \alpha\right)}{\alpha \pi_{1}}\right)+\frac{c \alpha \pi_{1}}{(1-\alpha)\left(\pi_{2}+c \alpha\right)}-c$. Therefore we can express each firm $i$ 's average promotional depth in the form of $\sup P_{i}-E p_{i}=\Phi_{i}(\alpha) \times c$, where $\Phi_{i}(\alpha)$ is some function of $\alpha$ only. This shows that the critical value $\alpha^{*}$ is independent of $c$. Solving $\Phi_{1}(\alpha)=\Phi_{2}(\alpha)$ numerically gives $\alpha^{*} \simeq 0.442$. Q.E.D.

\section{$7 \quad$ Reference}

Baye, Michael R. and John Morgan, "Information gatekeepers on the internet and the competitiveness of homogeneous product markets," American Economic Review, 91(3), 2001, 454-474.

Blattberg, Robert C. and Kenneth J. Wisniewski, "Price-induced patterns of competition," Marketing science, 8(4), 1989, 291-309.

Brynjolfsson, Eric and Michael Smith," Frictionless commerce? a comparison of internet and conventional retailers," Management Science, 46(4), 2000, 563-585.

Chen, Yuxin, Chakravarthi Narasimhan, Z. John Zhang, "Individual marketing with imperfect targetability," Marketing science, 20(1), 2001a, 23-41.

Chen, Yuxin, Chakravarthi Narasimhan, Z. John Zhang, "Consumer heterogeneity and competitive price-matching guarantees," Marketing science, 20(3), 2001b, 300-314.

Colombo, Richard A. and Donald G. Morrison, "A brand switching model with implications for marketing strategies," Marketing science, 8(1), 1989, 89-99.

Comanor, William S. and Thomas A. Wilson, "The effect of advertising on competition," Journal of Econometric Research, 1974, 18, 453-476. 
Dasgupta, Partha and Eric Maskin, "The existence of equilibrium in discontinuous economic games, I, II," Review of Economic Studies, 53, 1986, $1-41$.

Iyer, Ganesh and Amit Pazgal, "Internet shopping agents: Virtual colocation and competition," Marketing Science, 22(1), 2003, 85-106.

Jacoby, Jacob and Robert W. Chestnut, Brand Loyalty: Measurement and Management, John Wiley and Sons, 1978, New York, NY.

Kaul, Anil and Dick R. Wittink, "Empirical generalizations about the impact of advertising on price sensitivity and price," Marketing Science, 1995, 14, G151-160.

Lal, Rajiv and J. Miguel Villas-Boas, "Exclusive dealing and price promotions," Journal of Business, 69(2), 1996, 159-172.

Lal, Rajiv and J. Miguel Villas-Boas, "Price promotions and trade deals with multiproduct retailers," Management Science, 44(7), 1998, 935-949.

McGahan, A.M. and Pankaj Ghemawat, "Competition to retain customers," Marketing Science, 13(2), 1994, 165-176.

Mela, Carl, F., Sunil Gupta and Donald R. Lehmann, "The long-term impact of promotion and advertising on consumer brand choice," Journal of Marketing Research, 1997, 34, 248-261.

Mitra, Anusree and John G. Lynch, Jr., "Toward a reconciliation of market power and information theories of advertising effects on price elasticity," Journal of consumer research, 1995, 21, 644-659.

Moorthy, Sridhar, "Product and price competition in a duopoly," Marketing Science, 7(2), 1988, 141-168.

Narasimhan, Chakravarthi, "Competitive promotional strategies," Journal of Business, 61(4), 1988, 427-449.

Olson, Jerry C. and Jacob Jacoby, "A construct validation study of brand loyalty," Proceedings of the American Psychological Association, Vol. 6, 1971, 657-658.

Pessemier, Edgar A., "A new way to determine buying decisions," Journal 
of Marketing, 24, 1959, 41-46.

Png, I.P.L. and David Hirshleifer, "Price discrimination through offers to match price," Journal of Business, 60(3), 1987, 365-383.

Png, I.P.L. and David Reitman, "Why are some products branded and others not?" Journal of Law and Economics, 38, 1995, 207-224.

Raju, Jagmohan S., "A theory of price promotions," Unpublished doctoral dissertation, Graduate School of Business, Stanford University, Stanford, CA, 1988.

Raju, Jagmohan S., V. Srinivasan and Rajiv Lal, "The effects of brand loyalty on competitive price promotional strategies," Management Science, 36(3), 1990, 276-304.

Rao, Ram C., "Pricing and promotions in asymmetric duopolies," Marketing Science, 10(2), 1991, 131-144.

Rosenthal, Robert W., "A model in which an increase in the number of sellers leads to a higher price," Econometrica, 48(6), 1980, 1575-1579.

Salop, Steven and Joseph Stiglitz, "Bargains and ripoffs: A model of monopolistically competitive price dispersion," Review of Economic Studies, 44(3), 1977, 493-510.

Shaked, Avner and John Sutton, "Relaxing price competition through product differentiation," Review of Economic Studies, 49(1), 1982, 3-13.

Shilony, Yuval, "Mixed pricing in an oligopoly," Journal of Economic Theory, 14, 1977, 373-388.

Shum, Matthew, "Does advertising overcome brand loyalty? Evidence from the breakfast-cereals market," Journal of Economics and Management Strategy, 13(2), 2004, 241-272.

Simester, Duncan, "Note. Optimal promotion strategies: a demand-sided characterization," Management Science, 43(2), 1997, 251-256.

Varian, Hal, "A model of sales," American Economic Review, 70(4), 1980, 651-659. 